Aus dem Institut für Hygiene und Mikrobiologie

der Universität Würzburg

Vorstand: Prof. Dr. M. Frosch

\title{
The Role of Endosymbiotic Wolbachia Bacteria in the Pathogenesis or River Blindness
}

\author{
Inaugural - Dissertation \\ zur Erlangung der Doktorwürde der \\ Medizinischen Fakultät \\ der \\ Julius-Maximilians-Universität zu Würzburg \\ vorgelegt von \\ Amélie von Saint André - von Arnim \\ aus Boston
}

Würzburg, November 2007 
Referent: $\quad$ Prof. Dr. M. Frosch

Korreferent: $\quad$ Prof. Dr. M. Lutz

Dekan: Prof. Dr. Matthias Frosch

Tag der mündlichen Prüfung: 02.12.2008

Die Promovendin ist Ärztin 


\section{TABLE OF CONTENTS}

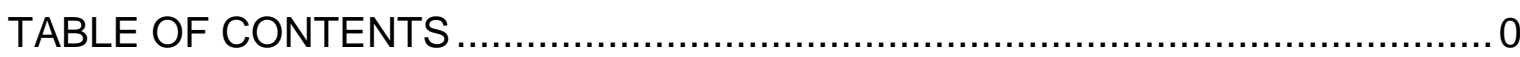

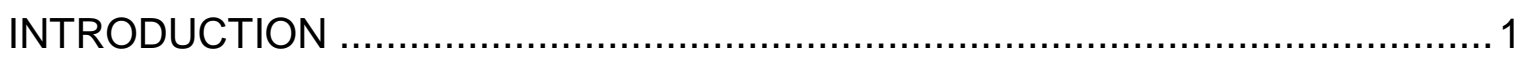

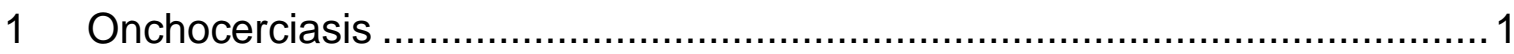

2 Wolbachia endosymbiotic bacteria.................................................... 4

3 Pathogenesis and host immune responses in onchocerca keratitis............... 5

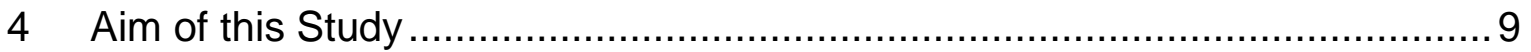

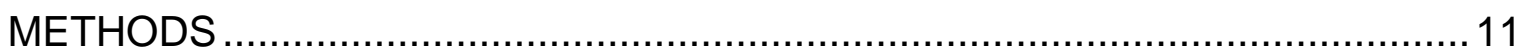

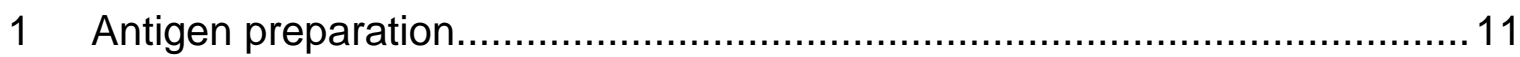

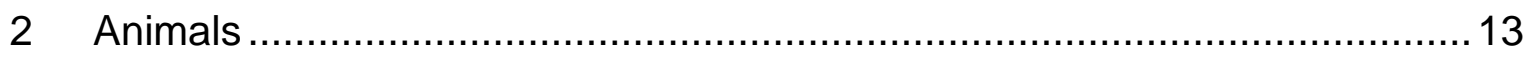

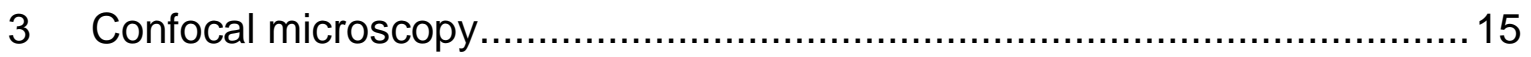

4 Immunohistochemical analysis of corneal inflammation .............................. 16

5 Detection of chemokines in the cornea ................................................. 17

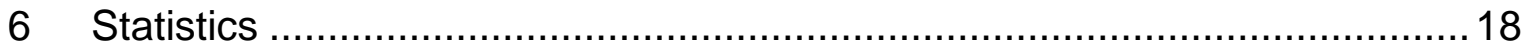

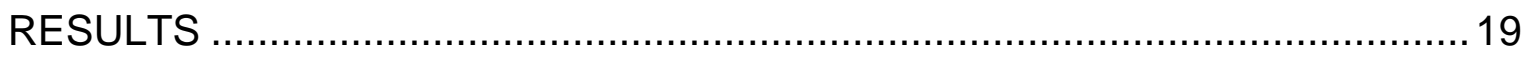

1 Doxycycline treated $O$. volvulus antigen has an impaired capacity to induce

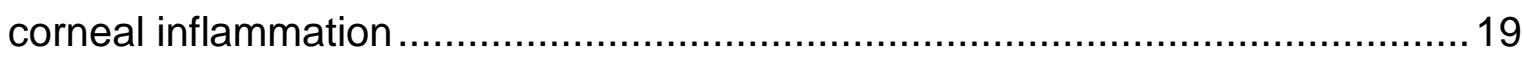

2 Filaria - induced inflammatory response is dependent on the presence of

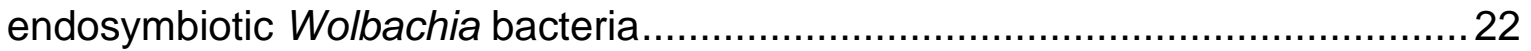

3 Toll - like receptor 4 dependence of $O$. volvulus keratitis ...........................24

4 TLR4 and Wolbachia dependent expression of PECAM-1 and of neutrophil

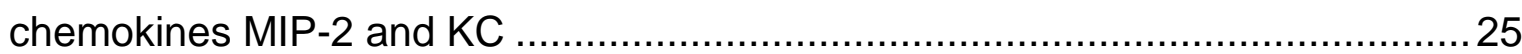

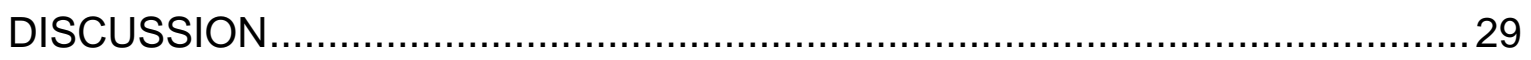

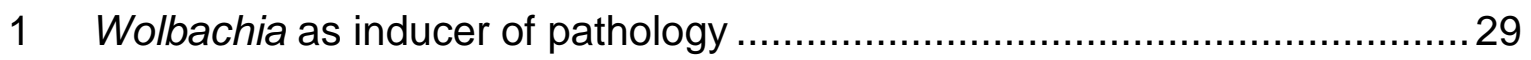

2 Wolbachia endobacteria - new targets for therapy ……............................. 35

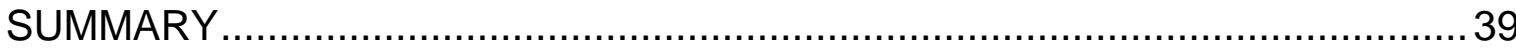

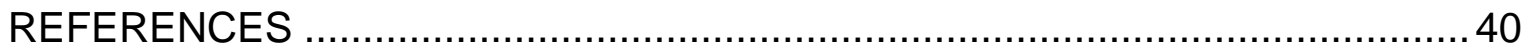





\section{INTRODUCTION}

\section{Onchocerciasis}

Onchocerciasis, or river blindness, is a parasitic worm disease caused by the filarial nematode Onchocerca volvulus. It affects 17.7 million people in Africa, Latin America, and Yemen and is the second most common cause of preventable blindness in sub-Saharan Africa [1]. Ocular disease is thought to result from a host-derived inflammatory reaction which occurs in response to the death of parasite microfilaria in the eye. Using a mouse model (Figure 1) for river blindness in which soluble extracts of filarial nematodes are injected into the corneal stroma,
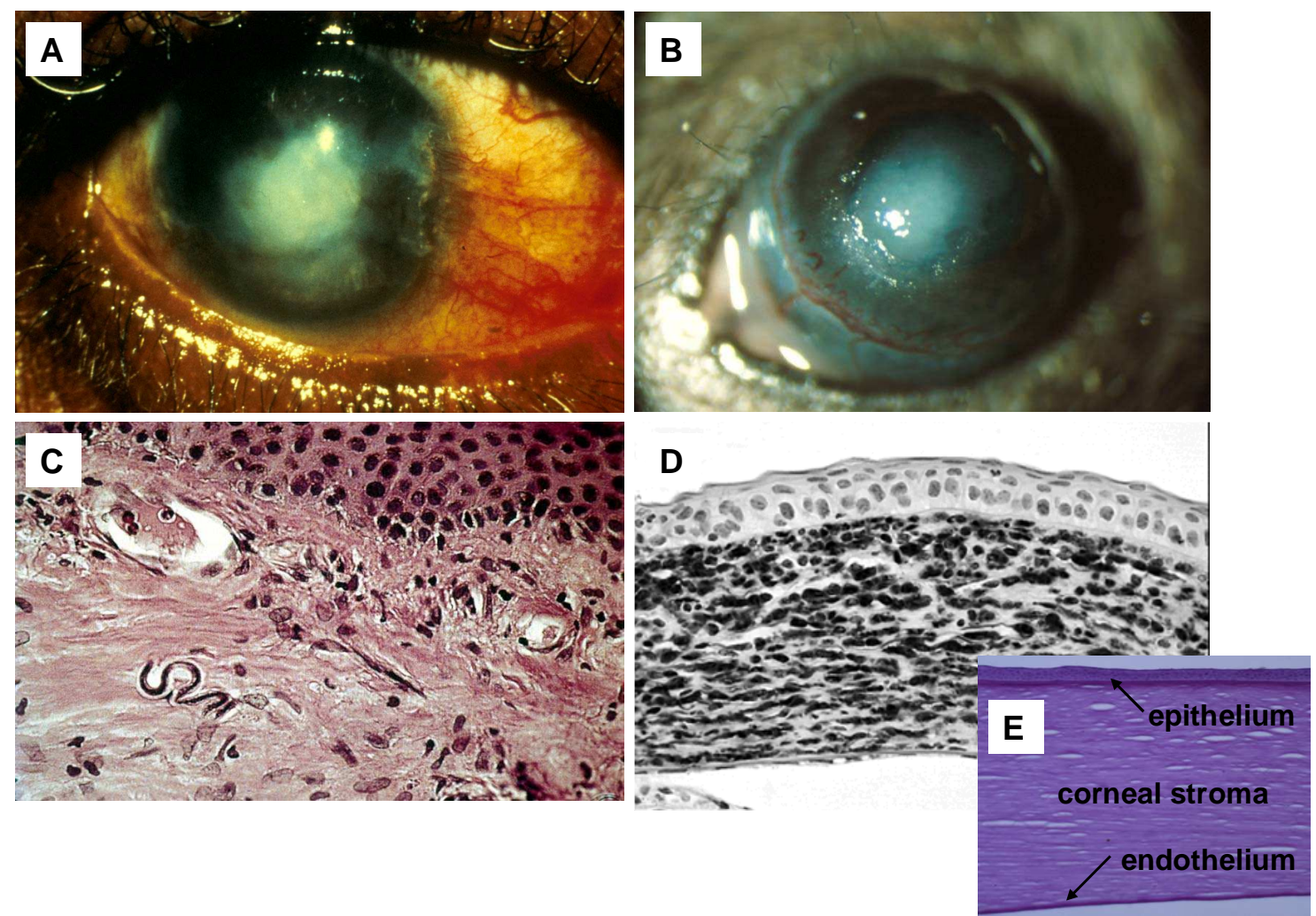

Figure 1 Mouse model of $O$. volvulus keratitis. Injection of $O$. volvulus antigens into the murine corneal stroma results in progressive corneal opacification and neovascularization (B), similar to human Onchocerca keratitis (A) (Photo by Dr. H. Taylor (University of Melbourne, Australia)) and (C) (Photo by D.H. Connor (University of Wisconsin, USA)) with stromal thickening and migration of inflammatory cells to the cornea. (D) demonstrates infiltration of neutrophils to the corneal stroma of a C57BL/6 mouse 24 hours after intracorneal injection of Wolbachia containing $O$. volvulus extract. The cornea was immunostained with anti-neutrophil antibody and neutrophils visualized by bright field microscopy (original magnification is $x 200$ ). (E) shows a normal murine cornea without inflammation. Images (D) and (E) by A. v. Saint André. 
this study investigates the role of the parasite's endosymbiotic Wolbachia bacteria in the pathogenesis of ocular onchocerciasis.

Onchocerciasis is transmitted by Simulium spp. blackflies, which transfer infective L3 stage larvae during a blood meal (Figure 2). These larvae mature to female and male worms within a year and are located in subcutaneous nodules (onchocercomas) within the human host. The numbers of female worms in infected people may range from 1 to 60 or more [2], each of them producing millions of microfilariae during their lifetime [3] which lasts 10 to 14 years. It is this microfilarial stage that is not only critical in the maintenance of the parasite's life cycle, but is the actual pathological agent of onchocerciasis - causing ocular and various forms of skin disease. Blindness - the most devastating disease manifestation - is caused by inflammation in the eye as a result of microfilariae migrating through the skin and conjunctiva into the cornea. Microfilariae dying and degenerating in the cornea lead to loss of corneal clarity and thereby visual im-

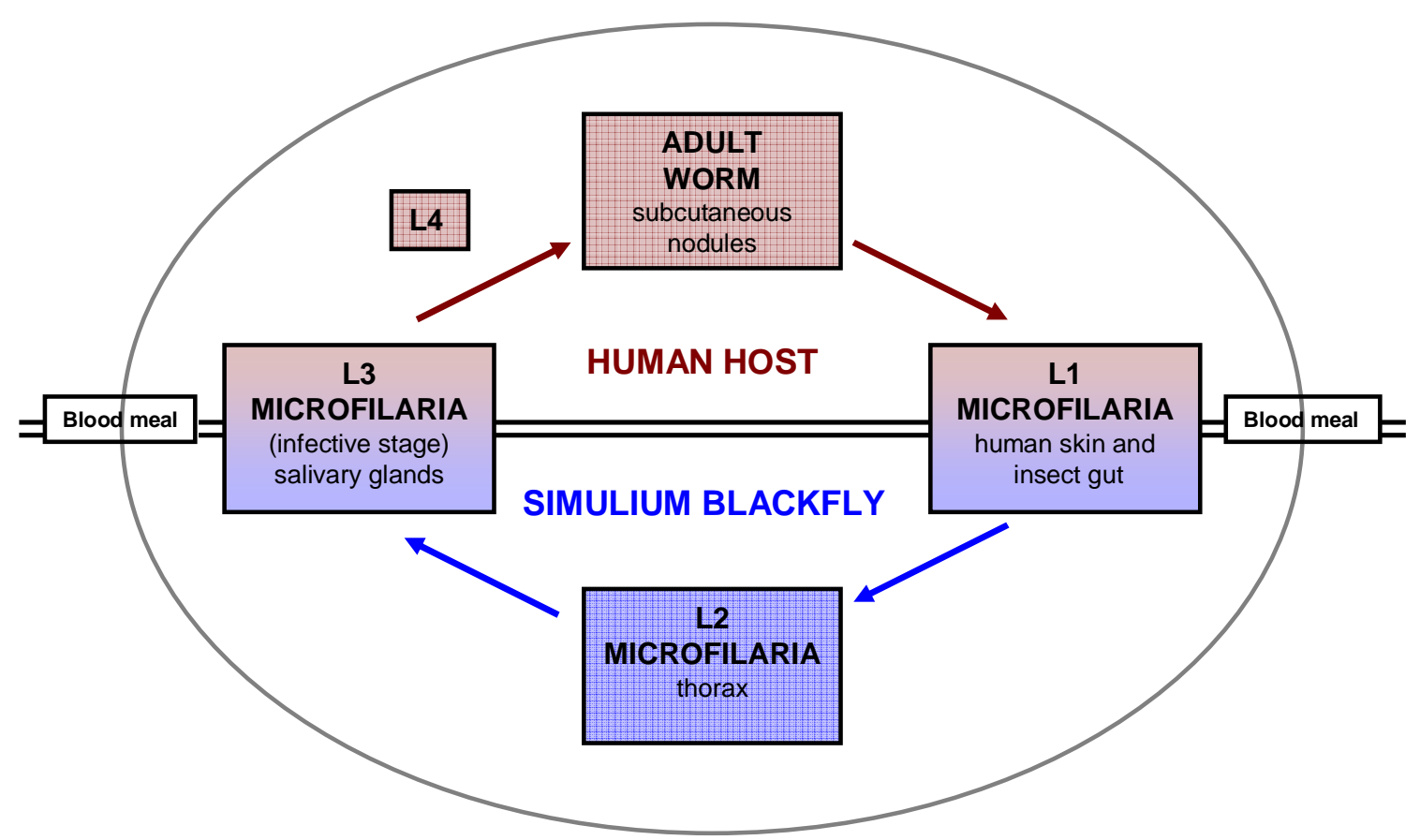

Figure 2 Transmission and Life Cycle of O.volvulus. The microfilaria is the most abundant stage, and it is critical in the maintenance of the parasite's life cycle, as well as being the pathological agent. Fertilized females release cyclically 1000-3000 microfilariae per day for the lifetime of the female. Microfilariae migrate through the human skin, and the parasite life cycle is continued after ingestion of microfilariae during the bloodmeal of a Simulium blackfly. Microfilariae undergo two molts in the blackfly, migrating through the insect gut, the thorax and into the salivary gland. On a subsequent blood meal, infective third stage larvae are transmitted to the next human host where they undergo a further two molts to become adult males and females. Image by A. von Saint André. 
pairment. This sclerosing keratitis may be followed by posterior chamber involvement including chorioretinitis and optic atrophy [4].

Major steps have been taken towards control of onchocerciasis, due to the successful joint efforts and support from WHO and other UN agencies, the World Bank, and a coalition of non-governmental and development organizations. These onchocerciasis control programs utilize a twofold strategy. Mass distribution of the microfilaricidal drug ivermectin is used as the principal intervention [5], and application of larvicides to the breeding sites of the mosquito vector has been used to interrupt transmission. The Onchocerciasis Control Program in West Africa (OCP) initially targeted the Simulium vector, later complemented by the distribution of ivermectin. This successful program ended in 2002. Two further programs, the Onchocerciasis Elimination Program of the Amerocas (OEPA) and the African Program of Onchocerciasis Control (APOC) have been implemented based on mass distribution of the macrofilaricidal drug ivermectin, either semiannual (OEPA) or annually (APOC). Recent endemicity studies, however, suggest that, at least in Africa, this approach will not stop transmission of disease and in areas where interruption of transmission has been achieved by vector control, the infection will be reimported by (re)migration of people who still carry the infection [6, 7]. Even after the end of transmission, mass treatment has to continue at least as long as adult worms survive in humans (up to 14 years) [8]. Therefore, the Conference on Eradicability of Onchocerciasis concluded that eradication was not feasible given the need of sustainable treatment at a coverage of $65 \%$ for 35 or more years [7]. Furthermore, should resistance to ivermectin - the only currently available drug - for human onchocerciasis arise, as an $O$. volvulus phenotype in Ghana with suboptimal drug response after repeated ivermectin treatments recently suggested $[9,10]$, control activities would be severely compromised. Thus new chemotherapeutic strategies need to be developed. However, long-standing research to develop a macrofilaricide - a drug that could kill or permanently sterilize the adult $O$. volvulus parasite - with qualities compatible with public health use, has to date, failed. 


\section{Wolbachia endosymbiotic bacteria}

It has been known since the mid-1970s that most filarial nematodes are infected with intracellular bacteria [11-13]. But it is only recently that the importance in the biology of these helminthes has been realized. Wolbachia are Rickettsia-like, matrilineally inherited [14], obligate intracellular bacteria that infect many species of invertebrates [15] and most filarial parasites of importance for human health, including O. volvulus, B. malayi, W. bancrofti and some Mansonella species. Three filarial parasite species, Acanthocheilonema viteae (infects rodents), Onchocerca flexuosa (infects deer) and Loa Loa $[16,17]$ do not naturally contain Wolbachia and are often used as experimental controls $[18,19]$ as in this study. Phylogenetic classification of Wolbachia is within the order of Rickettsiales, family Anaplasmataceae, which also contains the bacteria Ehrlichia, Anaplasma and Neorickettsia [20]. Presently there is a single valid species in the genus Wolbachia for both filariae and arthopods, which is Wolbachia pipientis [18, 2123]. Six major clades $(A-F)$ of Wolbachia have been identified to date [23, 24]: $A$, $B, E$, and $F$ have been reported from insects, arachnids, and crustaceans; $C$ and $D$ from filarial nematodes. The bacteria are found in all the stages of the nematodes' life cycle although they occur in varying proportions between individual worms and developmental stages [12, 25]. In adult nematodes, Wolbachia is concentrated in intracytoplasmatic vacuoles within the hypodermal lateral cords. In female worms, the bacteria are also present in the reproductive organs, including oocytes and developing embryonic stages in the uteri, whereas they have not been demonstrated in the male reproductive system [26-29] (see Figure 3).

Like other Rickettsia bacteria, Wolbachia are sensitive to the tetracycline class of antibiotics (tetracycline, doxycycline), and also to rifampicin and azithromycin [3037]. There are currently no technologies that enable long or short term culture of these endosymbionts in cell-free medium, i.e. outside the cytoplasm of invertebrate cell lines or intact filarial worms. Wolbachia from nematodes do not appear to infect host tissues, although bacterial DNA has been detected in filarialinfected individuals after diethylcarbamazine (DEC) treatment [38].

In arthropods Wolbachia have mostly parasitic habits manipulating arthropods' reproduction. In nematodes, in contrast, the endobacteria live as symbionts, in- 


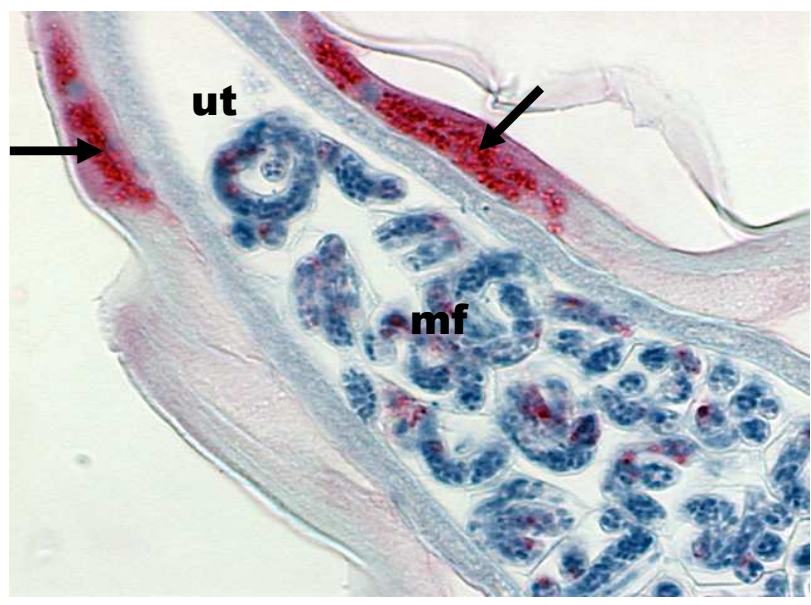

Figure 3 Wolbachia bacteria in section of female Brugia worm. Wolbachia bacteria (red) are concentrated in the hypodermal lateral cords (arrows) and around the uterus (ut) as well as within the microfilaria (mf) (original magnification $X$ 200). Adult worms were fixed in $4 \%$ formaldehyde, embedded in paraffin and sliced into $5 \mu \mathrm{M}$ sections followed by immunohistochemical staining using rabbit anti-recombinant wsp antisera and Vector red substrate. Image by Amy Hise (Case Western Reserve University, Cleveland, $\mathrm{OH}, \mathrm{USA}$ ).

creasing their own survival by increasing the fitness of the host. The long coevolution of the bacteria and the worm $[18,22]$ is expected to result in coadaptation and reciprocal dependence. Gene sequence analysis has provided a further indication that the association between Wolbachia and filarial nematodes is not parasitic [40, 41]. Although little is known about the specific molecular interaction between the symbiont and the host, Wolbachia appears to be essential for embryogenesis and larval development of the nematode and is, therefore, designated as obligatory symbiotic [19, 31, 32, 42]. As obligatory symbionts, Wolbachia can be used as a target for therapy.

\section{Pathogenesis and host immune responses in onchocerca keratitis}

The transparent nature of the mammalian cornea is due to the highly organized arrangement of collagen fibrils and a tightly regulated level of hydration, which is maintained by the resident cells. These include epithelial cells, which are the external barrier; stromal fibroblasts (keratocytes), which produce the collagen and proteoglycans that form the matrix of the stroma; and corneal endothelial cells, which maintain the hydration level of the cornea by pumping $\mathrm{H}_{2} \mathrm{O}$ from the stroma to the anterior chamber [43]. The cornea is a unique tissue for studying immunologically regulated inflammatory processes, since it is considered to be immune privileged: due to the lack of blood and lymphatic vessels, i.e. structures that provide the conduit for transportation of immunologic components into and 
out of the tissue, the cornea lacks direct access to the immune system. The normal cornea also lacks professional antigen presenting cells such as dendritic cells and macrophages that are resident in most other tissues. However, all of this immune apparatus is present in the limbus (Figure 4).
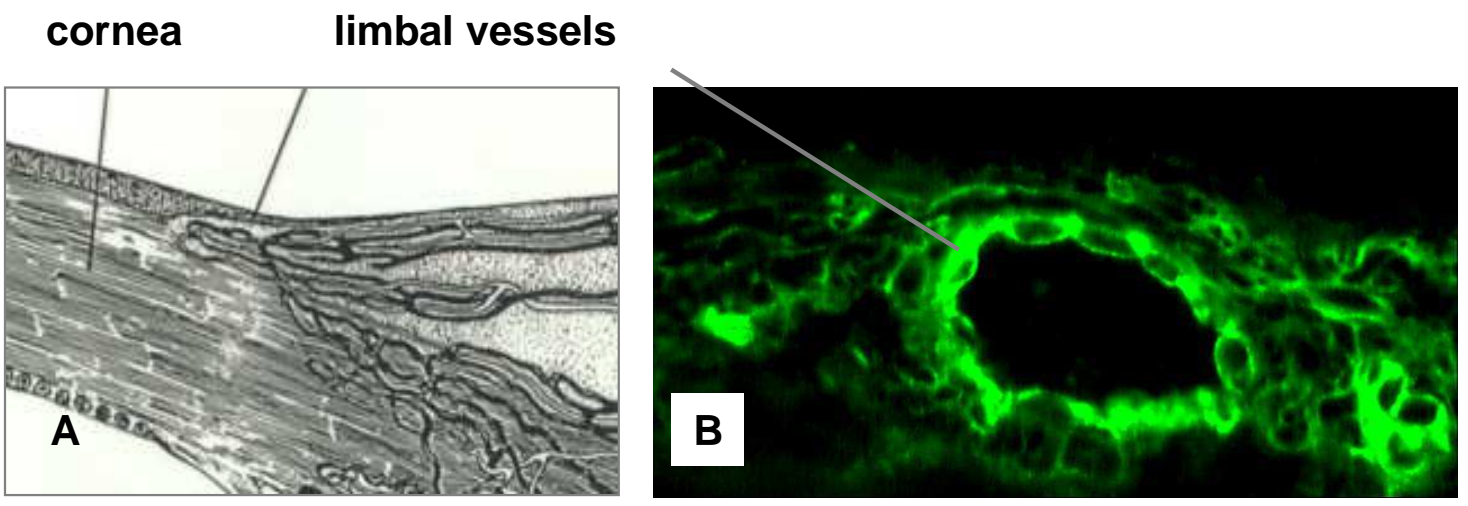

Figure 4 Recruitment of inflammatory cells from limbal vessels to the cornea. Since the normal cornea is avascular, leukocytes migrate from limbal vessels to the cornea. (A) Normal anatomy of the eye showing cornea and limbus (from "General Ophthalmology" 15th edition by D. Vaughan, T. Asbury, P. Riordan-Eva, Appleton \& Lange; 1999). (B) Image of limbal vessel stained for PECAM-1 (image by A. v. Saint André). Eyes of C57BL/6 mice were enucleated 24 hours after intrastromal injection of Wolbachia-containing $O$. volvulus antigen, showing upregulation of PECAM-1 which promotes neutrophil recruitment to the cornea. Original magnification $\times 400$.

During the course of inflammation, i.e. when microfilaria migrate to and degenerate in the cornea, leukocytes extravasate from blood vessels in the limbus, migrate to the center of the cornea and eventually this tissue becomes vascularized. Although inflammatory cells are important in host defense, they can also have cytotoxic effects on resident cells in the cornea, resulting in loss of corneal transparency, visual impairment, and blindness. Because the normal cornea is avascular and transparent, one can qualitatively and quantitatively observe the development of the inflammatory processes. The accessibility of the cornea and its relative separation from the immune system also facilitates local immunological manipulations without confounding system effects.

Previous studies in onchocerca keratitis focused on adaptive immune responses for at least two reasons: firstly, as a model for chronically infected individuals who are presumably sensitized prior to ocular involvement, there would be no example for innate immunity in the eye in the absence of an adaptive immune response, and secondly, experimental models showed no detectable corneal opacification or 
neovascularization with methods available at the time, unless animals were first immunized [44-46]. The understanding of the pathogenesis of onchocerca keratitis in a mouse model up to the point when this study was performed is summarized here (see Figure 5): the intrastromal injection of $O$. volvulus antigen is associated with a predominant CD4+, Th2 response both systemically and in the cornea, with $\lg \mathrm{E}$ and parasite specific $\lg \mathrm{G} 1$ being the predominant isotypes produced, and with the predominant early cellular infiltrate being neutrophils followed by a long-time mobilization of eosinophils 3 to14 days after exposure [44, 47-49]. The use of B cell-deficient $\mu \mathrm{MT}$ mice and FcyR-/- mice revealed that Fc receptors on neutrophils and eosinophils facilitate degranulation of these cells and disruption of corneal clarity [50,51]. Activation of resident corneal cells by breakdown products of microfilariae leads to secretion of proinflammatory cytokines, which stimulate synthesis of chemokines by keratocytes and induce the expression of adhesion molecules on vascular epithelial cells followed by recruitment of neutrophils and eosinophils: neutrophil recruitment is mediated by PECAM-1 and chemokine receptor CXCR2, whereas eosinophil recruitment is dependent on eotaxin, P-selectin and ICAM-1 [51-54]. In humans, CXCR2 is the receptor for IL-8 which is an important neutrophil attractant and activator during acute inflammation [55-57]. Mice, however, do not produce this chemokine but have two functional homologues, $\mathrm{KC}$ and MIP-2 that can bind to the murine CXCR2 receptor and are important mediators of neutrophil extravasation and recruitment to extravascular sites [58]. Both KC and MIP-2 are produced in murine models of $O$. volvulus keratitis $[53,59]$.

The high turnover rates of microfilariae $-20,000$ to 300,000 or more per day in hyperendemic areas [3] - cause a persistent release of $O$. volvulus and Wolbachia products. The exposure of these antigens to the host defense system can induce immune responses to filarial as well as endobacterial molecules.

While it is well documented that helminth infections are principally characterized by Th2-like immune responses, increasing evidence at the time of this study [6063] strengthened the notion that the innate immune system, neutrophil and Th1type responses - observed in filarial infections preferentially after microfilaricidal treatment - may depend on stimuli derived from Wolbachia endobacteria. 


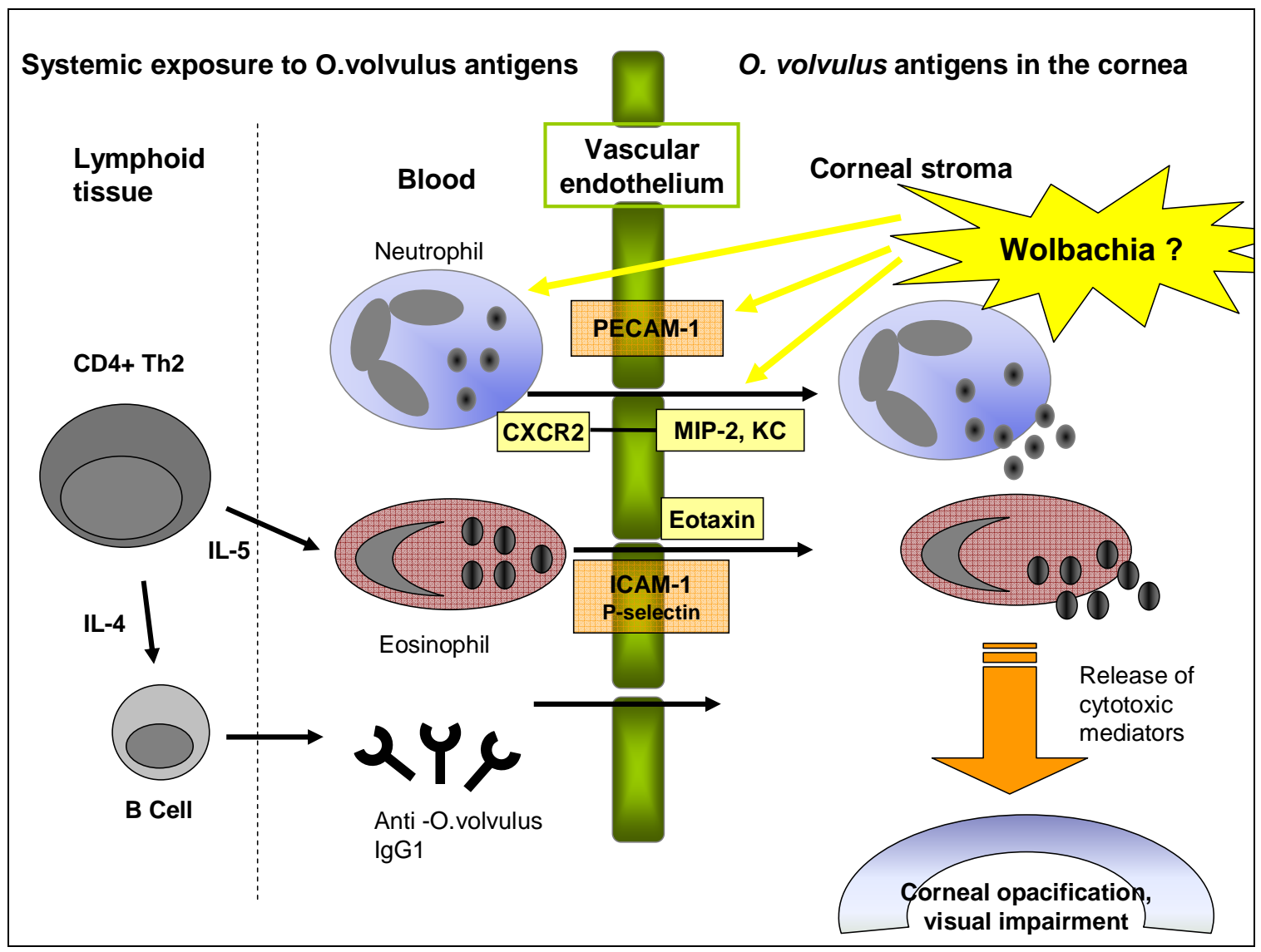

Figure 5 Proposed sequence of events in adaptive immune response underlying Onchocerca keratitis and possible role of Wolbachia in the pathogenesis of river blindness. Immunization or chronic infection induces a predominant Th2 response, with IL-4 leading to isotype switching to IgE and IgG1, and IL-5 inducing eosinophil differentiation; parasite antigens in the corneal stroma lead to activation of resident cells in the cornea, production of CXC and CC chemokines, elevated expression of adhesion molecules on vascular endothelial cells in the limbus, and biphasic infiltration of neutrophils and eosinophils to the corneal stroma. Immune complex-mediated cross linking of Fc receptors on neutrophils and eosinophils results in degranulation and release of cationic proteins and other cytotoxic mediators that disrupt normal corneal clarity, eventually leading to sclerosis and blindness. Image by A. v. Saint André, adapted from Kaifi et al.

The innate immune system in mammals senses the invasion of microorganisms using the family of Toll-like Receptors (TLRs), stimulation of which initiates a range of host defense mechanisms. TLR expression in the corneal epithelium has been confirmed. Initially and at time of these experiments, only TLR4 expression was reported [66], but recently other TLR including TLR2 have been found in the cornea [67-71] . Hence, this study investigates Wolbachia's role in activating the innate immune system by looking at neutrophil recruitment, TLR4, PECAM-1 and CXC chemokine dependent mechanisms in the development of onchocerca keratitis. 


\section{Aim of this Study}

This study aims to determine the role of Wolbachia endobacteria in the pathogenesis of $O$. volvulus keratitis. Using a murine model of corneal inflammation in which various parasite extracts are injected directly into the corneal stroma, corneas were examined by scanning confocal microscopy [72] and immunohistochemistry. Confocal microscopy enables in vivo measurement of stromal thickness and stromal haze, which are indicators of corneal edema and opacity, respectively, and can measure corneal abnormality responses that cannot be detected by slit lamp microscopy. The approach of characterizing Wolbachia's role in river blindness is threefold:

Firstly, soluble extracts of $O$. volvulus worms recovered from doxycycline treated individuals are compared with worms from untreated individuals and the corneal inflammatory responses are evaluated by confocal microscopy and immunohistochemical staining for neutrophils and eosinophils. According to immunohistochemistry and DNA analyses, $O$. volvulus worms from individuals treated with doxycycline have no detectable Wolbachia - as opposed to worms recovered from untreated individuals [31].

Secondly, the corneal effects to extracts from the rodent filaria Acanthocheilonema viteae, which naturally does not harbor Wolbachia are compared to extracts from endosymbiont containing Brugia malayi, a related filarial worm that causes lymphatic filariasis.

The third step determines if Wolbachia bacteria mediate corneal pathology by activating Toll-like Receptor (TLR) 4. TLR4 is one of the best characterized and one of the most important TLRs that mediates signals for a broad spectrum of ligands including heat shock proteins, fatty acids, LPS and oligosaccharides [73]. $\mathrm{C} 3 \mathrm{H} / \mathrm{HeJ}$ mice contain a genetic mutation that results in a truncated and inactive TLR4 protein [74]. Following injection of corneas with Wolbachia harboring $O$. volvulus antigen, corneal pathology in TLR4-mutant $\mathrm{C} 3 \mathrm{H} / \mathrm{HeJ}$ mice is compared to the wild-type $\mathrm{C} 3 \mathrm{H} / \mathrm{HeN}$ strain.

An additional step investigates further mechanisms of inflammatory infiltration to the cornea. $\mathrm{C} 3 \mathrm{H} / \mathrm{HeJ}$ and $\mathrm{C} 3 \mathrm{H} / \mathrm{HeN}$ mice are utilized to evaluate TLR4 dependent expression of the adhesion molecule PECAM-1, and production of the CXC cytokines MIP-2 and KC, all of which are essential for neutrophil recruitment to the 
cornea. Accordingly, PECAM-1 and KC expression is also investigated in C57BL/6 mice using O. volvulus antigen from doxycycline treated and untreated individuals to look at Wolbachia - dependent expression of these neutrophil attractant markers.

The aim of this study was to determine the role of Wolbachia in the pathogenesis of river blindness and thereby re-thinking our understanding of the immunopathology of filariasis, the adverse reactions to microfilaricidal drugs and new treatment options against filarial nematodes, which cause some of the most debilitating diseases in more than 150 million people [75-77] throughout the world's poorest communities. 


\section{METHODS}

\section{Antigen preparation}

\section{Doxycycline treated and non-treated $O$. volvulus antigen}

$O$. volvulus worms containing Wolbachia were recovered from infected individuals in Ghana who were either untreated (OvAg) or had been treated with doxycycline (OvAg/doxy) [31]. Phosphate-buffered saline (PBS) soluble parasite extracts were prepared by grinding, ultrasonication and centrifugation at $10,000 \mathrm{Xg}$ for 30 minutes. The total concentration was determined by standard Bradford assay [78] (Bio-Rad, Richmond, CA, USA). All materials used for the production of parasite products were sterilized and procedures were performed with strict aseptic techniques. Endotoxin levels in parasite extracts were detected by a sensitive chromogenic Limulus amebocyte lysate (LAL) test (BioWhittaker, Walkersville, MD, USA) with a quantifying LAL-activity between 0.1 to 1.0 endotoxin per $\mathrm{ml}$ using several dilutions of the extracts [61].

The effect of doxycycline on reducing Wolbachia numbers in the worms was demonstrated by immunohistochemistry using antibodies to bacterial heat shock protein 60 (hsp60) [31]. Soluble extracts were frozen at $-80^{\circ} \mathrm{C}$ until required. These parasite extracts were kindly provided by Achim Hoerauf and Norbert W. Brattig (Bernhard Nocht Institute for Tropical Medicine, Hamburg, Germany).

\section{O. volvulus antigen}

$O$. volvulus worms were recovered from subcutaneous nodules that had been surgically removed from infected patients in Cameroon, and kindly sent to us by Dr. Janet Bradley (University of Nottingham, Nottingham, U.K.). Parasites were recovered after digestion with collagenase (Sigma, St. Louis, MO, USA) as described elsewhere [44], and homogenized in Hanks' Balanced Salt Solution (HBSS) using a mortar and pestle. Insoluble material was removed by centrifugation, and the concentration of protein was determined by a commercial adaptation of the Bradford [78] method (Bio-Rad). All procedures were performed with strict aseptic techniques and endotoxin-free materials. Endotoxin levels in 
parasites were detected by quantitative LAL testing (BioWhittaker) using several dilutions of the extracts and quantifying LAL-activity between 0.1-1.0 endotoxin per $\mathrm{ml}$.

\section{B. malayi antigen}

Adult worms of the filarial parasite B. malayi, obtained live from the NIH filaria repository and shipped in sterile RPMI-1640 were processed by washing extensively with sterile PBS, then culturing for 7 days in RPMI, 5\% low endotoxin FCS and $1 \%$ penicillin/ streptomycin at $37^{\circ} \mathrm{C}$ in $5 \% \mathrm{CO}_{2}$. Daily media changes were performed to ensure that the final filarial extracts were free from contaminating bacterial products or endotoxin. After rinsing six times with cold sterile PBS, the worms were processed in cold RPMI by homogenization using endotoxin-free coarse glass tissue grinders followed by external sonication on ice. The filarial extracts were then centrifuged at $1500 \mathrm{rpm}$ for 15 minutes at $4^{\circ} \mathrm{C}$ and the supernatant was collected and frozen at $-20^{\circ} \mathrm{C}$. All procedures were performed using strict aseptic techniques and endotoxin-free materials. Quantitative LAL testing (BioWhittaker) was used to monitor culture media during the processing. Protein concentrations were determined using a commercial adaptation of the Bradford [78] method (Bio-Rad).

B. malayi extracts were kindly provided by Amy G. Hise (Case Western Reserve University, Cleveland, $\mathrm{OH}, \mathrm{USA}$ ).

\section{A. viteae antigen}

Adult $A$. viteae worms which naturally do not harbor Wolbachia were obtained from subcutaneous tissues of Mongolian gerbils. Adult male and female worms were collected under aseptic conditions and washed four times in RPMI-1640 containing 5\% FCS and 1\% penicillin/ streptomycin (Gibco Brl, Gaithersburg, MD, USA). Parasites were cultured at $37^{\circ} \mathrm{C}$ in $5 \% \mathrm{CO}_{2}$ for 5 days to ensure the absence of contaminating microorganisms and to select viable motile parasites. Adult parasites were separated into male and female worms under sterile conditions and washed four times in sterile PBS. Extracts were prepared by finely 
mincing the worms followed by homogenization on ice. All procedures were carried out under stringent sterile conditions with endotoxin-free materials. Extracts were centrifuged at $20,000 \mathrm{~g}$ for 30 minutes, and the supernatant was collected and stored at $-20^{\circ} \mathrm{C}$ until required. Protein concentration of parasite extracts was determined by the Coomassie protein assay (Pierce Chemical Co., Rockford IL, USA) [60].

A. viteae extracts were kindly supplied by Mark Taylor (Liverpool School of Tropical Medicine, Liverpool, U.K.).

\section{Animals}

6 to 8 week-old female $\mathrm{C} 57 \mathrm{BL} / 6$ and $\mathrm{C} 3 \mathrm{H} / \mathrm{HeN}$ mice were obtained from The Jackson Laboratory (Bar Harbor, ME, USA) and 6 to 8 week-old female $\mathrm{C} 3 \mathrm{H} / \mathrm{HeJ}$ mice from Harlan Sprague (Indianapolis, IN, USA). All mice were treated in accordance with the ARVO (Association for Research in Vision and Ophthalmology) Statement for the "Use of Animals in Ophthalmic and Vision Research".

\section{Injections into the corneal stroma}

Mice were anaesthetized with an intraperitoneal injection of $200 \mu \mathrm{l}$ of a $1.2 \%$ solution of 2,2,2-tribromoethanol (Aldrich, Milwaukee, WI, USA) containing 2.5\% 2-methyl-2-butanol (tertiary amyl alcohol) (Aldrich) dissolved in distilled water. Using a sterile 30-gauge needle, the corneal epithelial layer was abraded, and 1 $\mu \mathrm{g}$ of extract in $5 \mu \mathrm{l}$ of saline was directly injected into the corneal stroma using a 33-gauge needle attached to a Hamilton syringe (Hamilton, Reno, NV, USA).

In previous experiments the appropriate dosage for intracorneal injections had been made dependent on the extract's protein concentration. Comparing the inflammatory responses in murine corneas 24 hours after injection of $2 \mathrm{mg} / \mathrm{ml}$ versus $1 \mathrm{mg} / \mathrm{ml}$ of the different extracts, two out of four corneas which had received the higher dose of each antigen turned completely opaque and impossible to examine by confocal microscopy and one animal died (unpublished 
data). All the mice injected with the lower dose of extract survived and corneas were easily evaluated. All experiments were therefore performed using parasite extracts of $1 \mathrm{mg} / \mathrm{ml}$ of protein concentration.

Inflammatory responses to different parasite antigens were compared in pairs by injecting one of the matched up extracts into the left and one in the right eye of the same animal (see Figure 6). This set up of experiments excludes variation of host reactivity in different mice as a cause for altered reactions to the various parasite extracts.

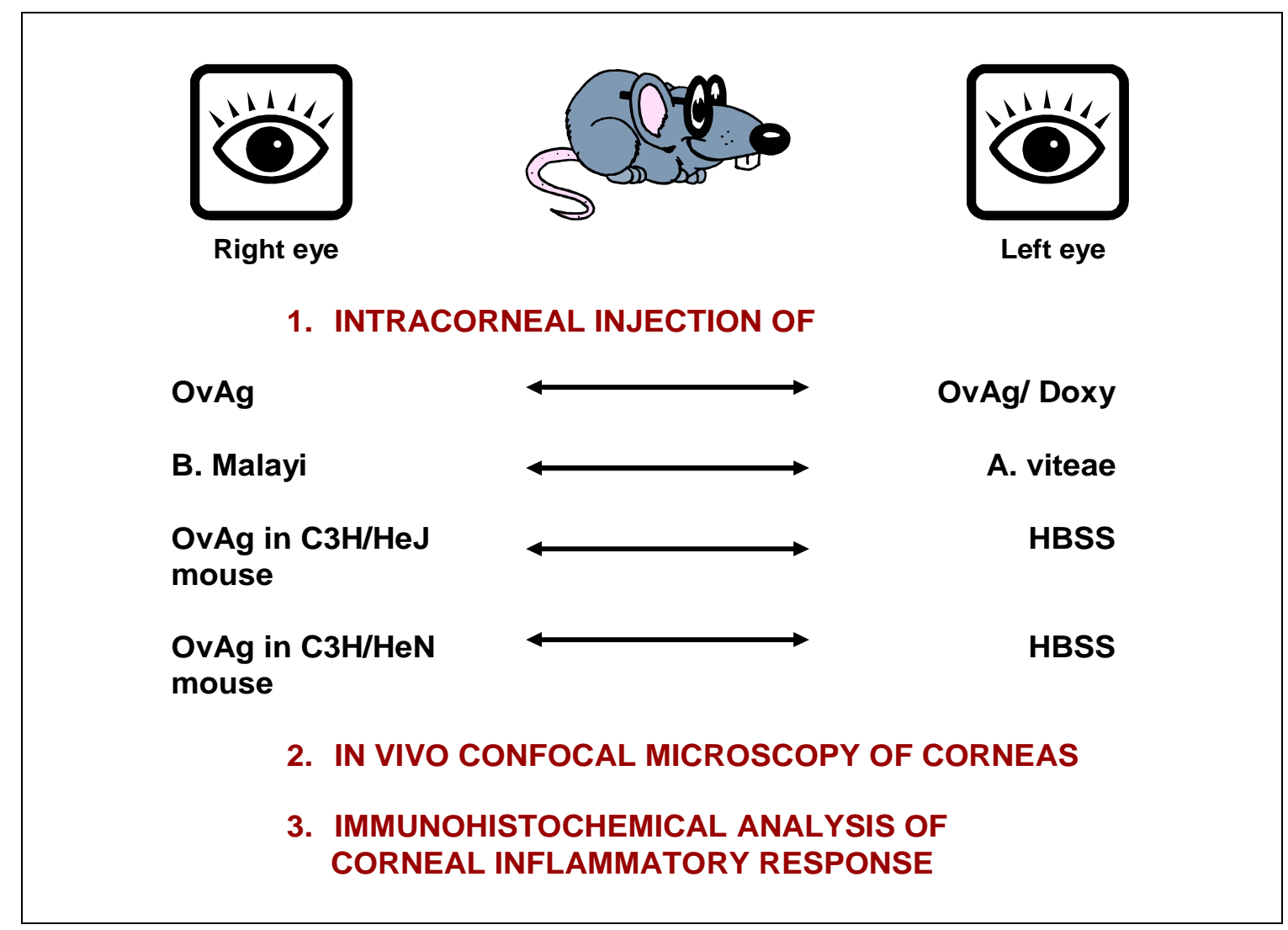

Figure 6 Basic set up and steps of experiments. Inflammatory reactions to different antigens were compared in the same animal by injecting one extract in the right, one in the left eye. In a second step corneas were examined by confocal microscopy, then mice were sacrificed and corneas analyzed immunohistochemically.

\section{Immunizations}

For the first set of experiment animals were given three weekly subcutaneous immunizations with $10 \mu \mathrm{l}$ of $\mathrm{O}$. volvulus antigens in a 1:1 ratio with adjuvant containing 10\% squalene (Aldrich), 0.4\% Tween Fisher (Fisher, Fair Lawn, NJ, USA), and 1\% pluronic acid (BASF Bioresearch, Parsippany, NJ, USA). 
The mouse model of $O$. volvulus keratitis had been established with a focus on a Th2 type response. Therefore weekly immunizations of mice had been performed in previous projects in order to stimulate the adaptive immune system. Since this study concentrated on the innate immune response, mice in all but the first experiment received intracorneal injections only without prior immunizations.

\section{Confocal microscopy}

In vivo examinations were performed using a scanning confocal microscope (Tandem scanning, Reston, VA, USA). The technique used was Confocal Microscopy through Focusing (CMTF). For this the mice were anesthetized as described previously. A drop of $2.5 \%$ hydroxypropyl methylcellulose was then

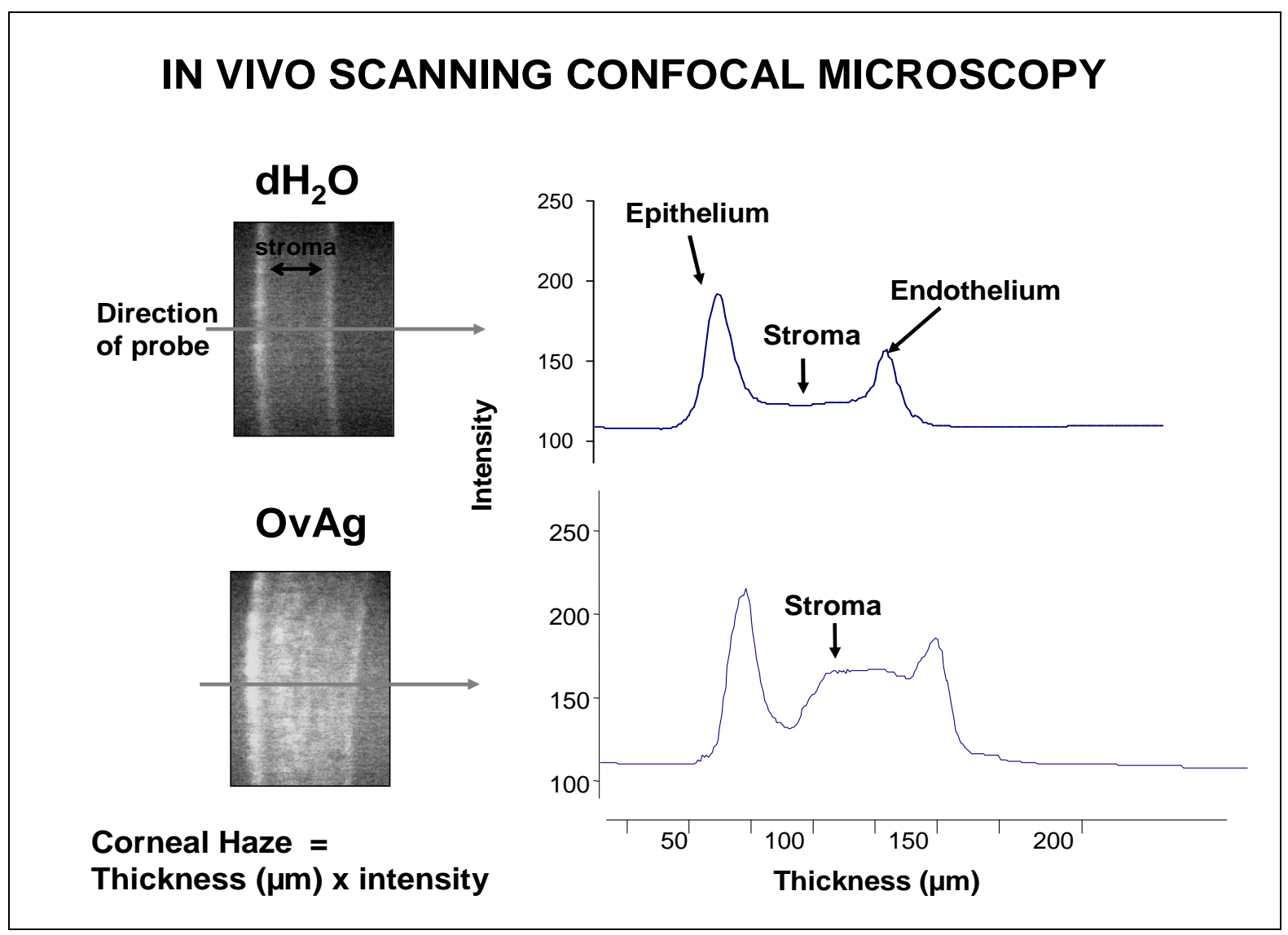

Figure 7 Confocal microscopy through focusing to measure stromal thickness and stromal haze of murine corneas. Injection of $O$. volvulus extract (OvAg) into the cornea (lower part) of C57BL/6 mice results in increased stromal thickness on both the CMTF derived image (left side) and intensity profile (right side), compared to injection of sterile water (upper part). Image by A. von Saint André. 
applied to the objective tip to eliminate the bright field reflections and the objective was brought in contact with the mouse eye. Keeping the gain constant in all the readings and using double frame rate (60 frames/ sec) and lens speed of 160 $\mu \mathrm{m} / \mathrm{sec}$, the images and intensity profiles were obtained. From these profiles, the stromal thickness as well as the stromal haze were calculated [72] (Figure 7). CMTF was used to evaluate murine corneas 24 hours after intrastromal injection of parasite antigen.

\section{Immunohistochemical analysis of corneal inflammation}

\section{Detection of Neutrophils and Eosinophils}

For immunohistochemical staining, enucleated eyes were snap frozen in liquid nitrogen, stored at $-70^{\circ} \mathrm{C}$, and $5 \mu \mathrm{m}$ sections were fixed in $4 \%$ formaldehyde for $25 \mathrm{~min}$, then rinsed in PBS (Sigma) and covered with proteinase $\mathrm{K}$ (Dako, Carpinteria, CA, USA) for 8 minutes to improve accessibility of antibodies to target sites within the tissue, since proteolytic digestion by proteinase $\mathrm{K}$ exposes certain epitopes which may have been masked during formalin fixation. Neutrophils were detected by incubating slides for two hours with the rat anti-mouse antibody NIMP-R/14 (kindly supplied by Achim Hoerauf, Bernhard Nocht Institute of Tropical Medicine, Hamburg, Germany), diluted 1:100, followed by fluorescein isothiocyante (FITC)-conjugated rabbit anti-rat IgG (Caltag Laboratories, Burlingame, CA, USA) diluted 1:200 as secondary antibody for 45 minutes. Eosinophils were immunostained with rabbit antisera to eosinophil major basic protein (MBP, kindly provided by Jamie Lee, Mayo Clinic, Scottsdale, AZ, USA), diluted 1:5000. FITC-labeled goat anti-rabbit IgG (Caltag Laboratories), diluted 1:200, was used as a secondary antibody and incubated for 45 minutes. Stained sections were washed in PBS and coverslipped with Vectashield Mounting Medium (Vector Laboratories, Burlingame, CA, USA) to inhibit quenching. Positively stained cells were examined by fluorescence microscopy. Cells were counted either throughout the corneal section or in representative fields at $600 \mathrm{x}$ magnification. 


\section{Detection of PECAM-1}

Eyes were snap-frozen in liquid nitrogen, stored at $-70^{\circ} \mathrm{C}$, and $5 \mu \mathrm{m}$ sections were fixed for 10 minutes in $-20^{\circ} \mathrm{C}$ acetone. Slides were air-dried, then rehydrated in PBS (pH 7.4) and stained with rat anti-mouse IgG against PECAM-1 (MEC 13.3, PharMingen, San Diego, CA, USA). Primary antibodies were diluted 1:100 in PBS containing $1 \%$ fetal calf serum and incubated for two hours at room temperature. FITC-labeled rabbit anti-rat IgG $(\mathrm{H}+\mathrm{L})$ (Caltag Laboratories) diluted 1:200 was used as a secondary antibody and incubated for 45 minutes. Stained sections were washed in PBS, coverslipped with Vectashield Mounting Medium (Vector Laboratories) and examined by fluorescence microscopy.

\section{Evaluation of Staining Intensity}

Evaluation of expression of FITC-stained adhesion molecules on limbal vessels was based on the method described by Tang and Hendricks [79] for detection of PECAM expression. After immunostaining for PECAM-1, images of limbal vessels were captured using a digital camera model DC330 (PAGE-MTI Inc., Michigan City, IN, USA) and Scion Image Software (Version 1.62c; National Institutes of Health, Bethesda, MD, USA, modified by Scion Corporation). To evaluate the relative fluorescence intensity, the mean brightness value of the green channel of the three most intensely stained vessel areas was determined using Adobe Photoshop 5.0 (Adobe Systems Inc., San Jose, CA, USA) with a set 400 pixel square area. Four vessels from each eye were analyzed, the background reading in unstained areas of the cornea was subtracted from these values, and the mean plus/ minus standard error of the fluorescence intensity for each vessel was estimated. Data was then presented as the percent maximum value for the antibody.

\section{Detection of chemokines in the cornea}

To determine the concentration of the chemokines MIP-2 and KC in murine corneas, animals were sacrificed and corneas were carefully dissected to avoid 
removing surrounding conjunctival tissue and underlying iris. The corneas were

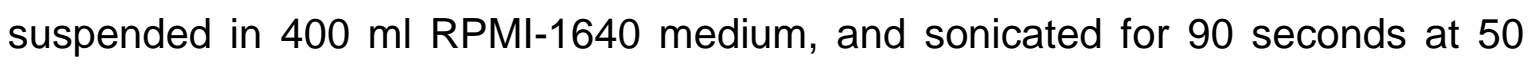
cycles per second (Sonics VibraCell, Danbury, CT, USA). After centrifugation, the concentrations of MIP-2 and KC were detected in supernatants by two-site ELISA following the manufacturer's directions (R\&D Systems, Minneapolis, MN, USA). The limit of detection for MIP-2 was $1.5 \mathrm{pg} / \mathrm{ml}$, and less than $2.0 \mathrm{pg} / \mathrm{ml}$ for KC.

\section{Statistics}

Statistical significance was determined using an unpaired Student's $t$ test (Prism Graph Pad Software, San Diego, CA, USA). A value of $p<0.05$ was considered to be significant. 


\section{RESULTS}

\section{Doxycycline treated 0 . volvulus antigen has an impaired capacity to induce corneal inflammation}

This first set of experiments compares the inflammatory infiltrate after exposure of C57BL/6 mice to O. volvulus extracts originating from onchocerciasis patients either with (OvAg/doxy) or without (OvAg) prior doxycycline treatment. As previous studies had shown, inflammatory cells infiltrate the cornea in a biphasic manner, with neutrophils predominant in the first 24 hours after intrastromal injection and their replacement by eosinophils after 72 hours [51, 53, 64]. Consistent with this observation, Figure 8 shows neutrophil recruitment peaking at 24 hours in eyes injected with OvAg. However, the number of these cells was significantly reduced in corneas treated with OvAg/doxy $(p=0.018)$, i.e. without Wolbachia. Eosinophil mobilization to the cornea increased three days after exposure to parasite antigens, however their number was the same for the two different extracts. Neutrophil, but not eosinophil infiltration therefore seems to be dependent on the presence of Wolbachia endobacteria.

LAL testing revealed an endotoxin level of $3.83 \mathrm{EU} / \mathrm{ml}$ for untreated OvAg and $1.98 \mathrm{EU} / \mathrm{ml}$ for OvAg/doxy extracts of the same protein concentration, therefore
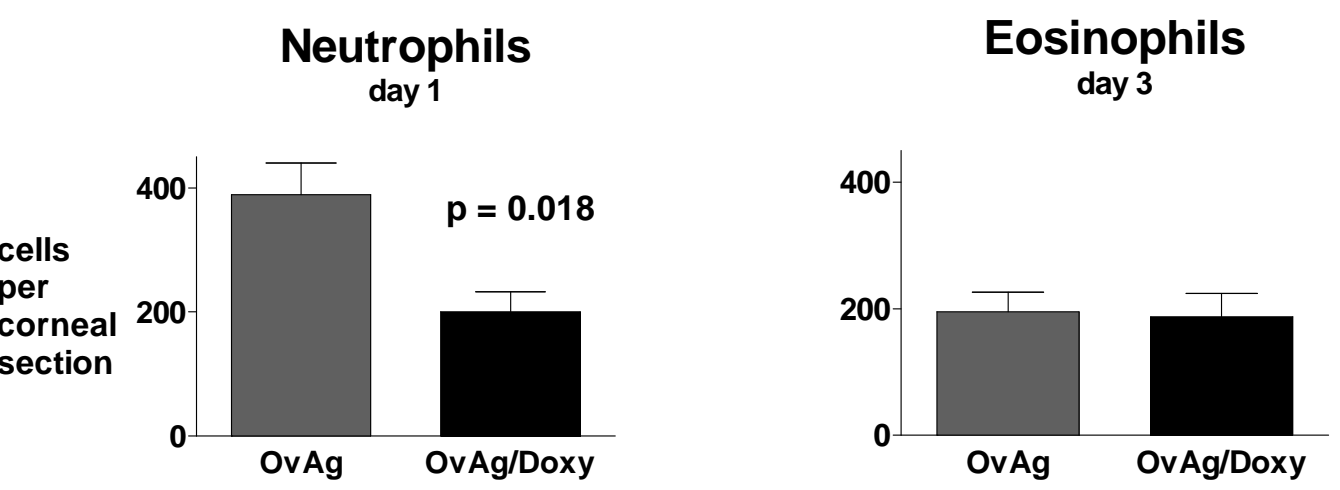

Figure 8 Diminished neutrophil recruitment to the cornea 24 hours after injection of 0 . volvulus worms derived from doxycycline treated individuals. Untreated Wolbachiacontaining OvAg revealed a significantly higher neutrophil infiltration $(p=0.018)$, indicating that Wolbachia bacteria may contribute to the pathogenesis of river-blindness. Eosinophil numbers remained the same for both treated and untreated $O$. volvulus extracts 72 hours post intrastromal injection. 
suggesting a small amount of endotoxin contamination of both extracts. The increased endotoxin level of untreated OvAg was at the time when this experiment was done interpreted as inherent Wolbachia - LPS. Recent genome sequencing of Wolbachia, however, has shown that the endosymbiont lacks the genes required for LPS biosynthesis [40, 80]. Although endotoxin levels in these two extracts are low and not of major difference, some degree of endotoxin related effect on neutrophil recruitment cannot be excluded in this experiment.

O. volvulus extracts from doxycycline treated worms (i.e. not containing
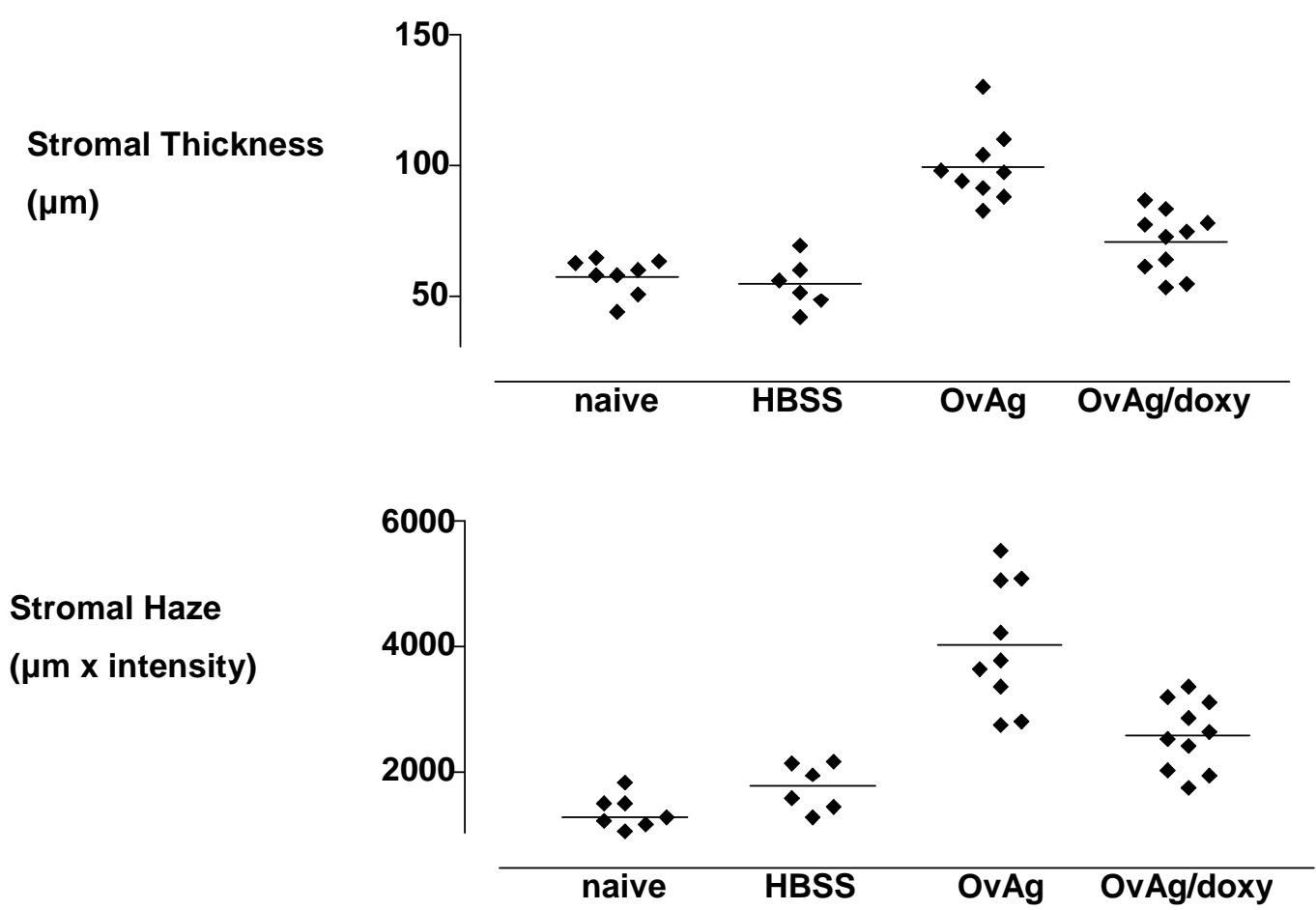

Neutrophils

(per $5 \mu \mathrm{m}$ section)

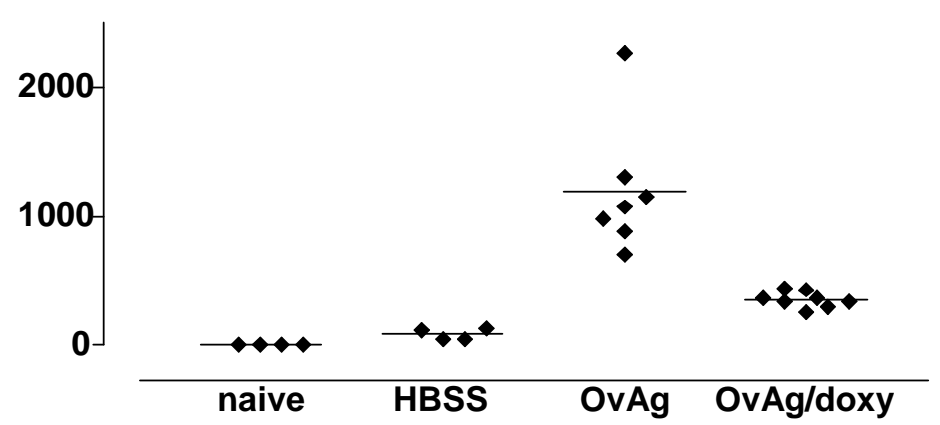

Figure 9 Diminished stromal thickness, stromal haze and neutrophil recruitment in corneas injected with doxycycline-treated and therefore Wolbachia-free 0 . volvulus extracts (OvAg/doxy). Untreated OvAg extracts led to a significantly higher inflammatory response. Data points represent individual corneas from a single experiment. 
Wolbachia) induced significantly lower stromal thickness $(p=0.0001)$, stromal haze $(p=0.0012)$ and neutrophil infiltration $(p=0.0005)$ than untreated worms (i.e. containing Wolbachia bacteria) 24 hours after intracorneal injection (Figure 9). Although minor compared to untreated worms, extracts from treated worms also induced responses that were higher than naïve corneas or corneas injected with saline $(p<0.0001$ for neutrophil infiltration, $p=0.0129$ for stromal thickness and $p=0.0071$ for stromal haze), indicating that not only Wolbachia, but also filarial antigens contribute to the inflammatory reaction. Again, the small endotoxin content of OvAg and OvAg/Doxy may also contribute to this effect. Stromal thickness and stromal haze values for saline-injected mice were similar to naïve mice, meaning that the trauma of the injection has no or little effect on these parameters. This experiment was repeated four times showing similar results with both immunized and unimmunized mice. Figure 10 shows the typical confocal microscopy images obtained in these experiments.

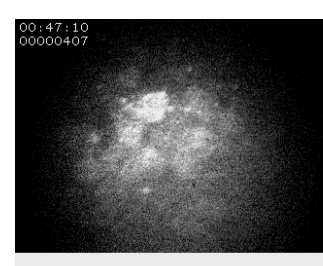

A
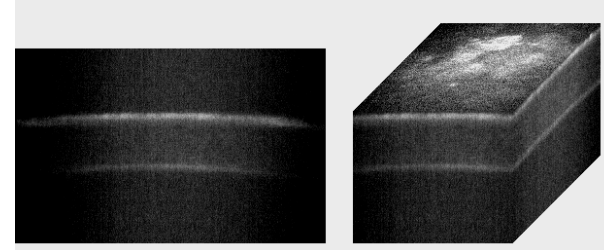

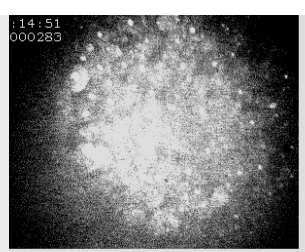

B

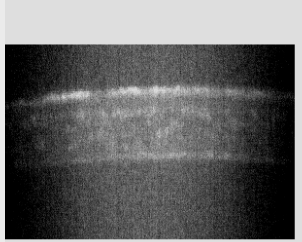

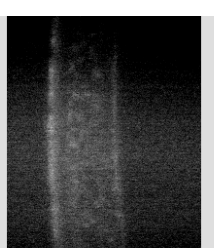

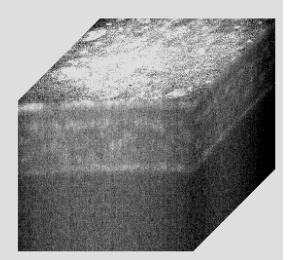

Figure 10 Confocal microscopy images of B57BL/6 mice 24 hours after injection of either saline (A) or untreated Wolbachia-containing $O$. volvulus extract (B). Stromal thickness (top right and bottom left of both $A$ and $B$ ) and cell infiltration are increased in corneas exposed to parasite extract. Greater cell infiltrate can be seen especially in the corneal epithelial layer of OvAg-injected corneas (top left corner of B) compared to saline-treated eyes (top left corner of A). Images by $A$. von Saint André. 


\section{Filaria - induced inflammatory response is dependent on the presence of endosymbiotic Wolbachia bacteria}

The inflammatory response to extracts of Brugia malayi, a filarial worm that contains Wolbachia, was compared to extracts from the rodent filaria Acanthocheilonema viteae, which naturally does not harbor the endosymbiotic bacteria. Corneas of B57BL/6 mice were injected with these extracts, and the inflammatory response was measured as described above. Figure 11 shows that injection of $B$. malayi extract stimulates a pronounced neutrophil infiltration, with elevated stromal haze and stromal thickness, similar to the untreated OvAg

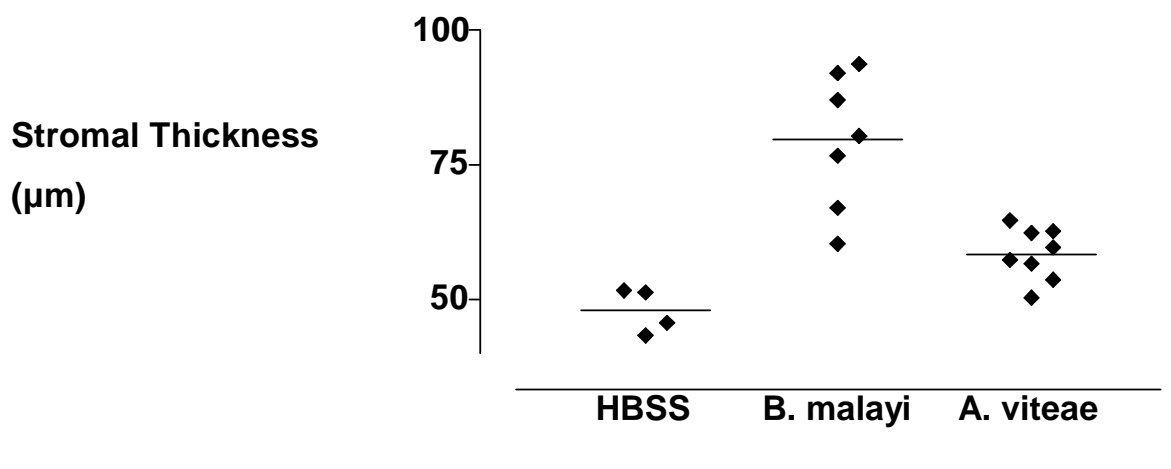

Stromal Haze
$(\mu \mathrm{m} x$ intensity)

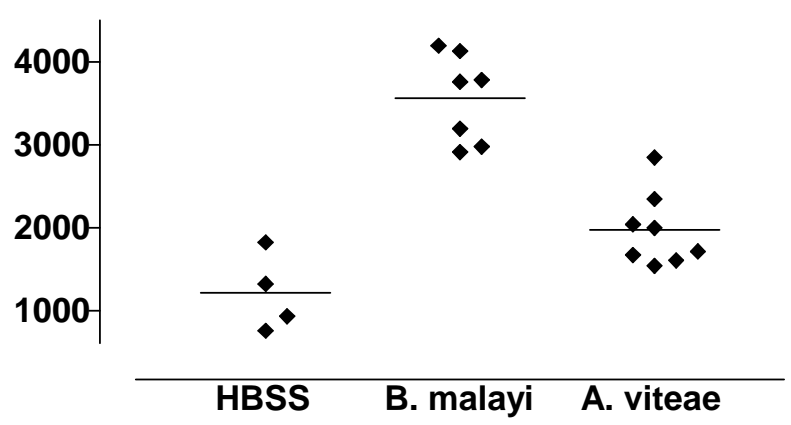

Neutrophils

(per $5 \mu \mathrm{m}$ section)

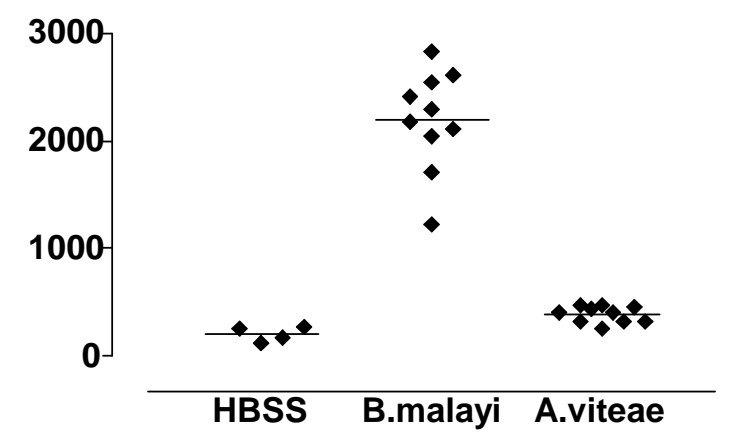

Figure 11 Significantly increased stromal thickness, stromal haze and neutrophil recruitment for Wolbachia containing $B$. malayi extracts compared to endosymbiont-free $A$. viteae extracts. Data points represent individual corneas from a single experiment. 
response. In contrast, the inflammatory response to $A$. viteae extract was significantly lower compared to the reaction to $B$. malayi extracts (Figure 4) with $p$ $=0.0007$ for stromal thickness, $p<0.0001$ for stromal haze and $p<0.0001$ for neutrophil infiltration. Similar results were obtained by a repeat experiment. The immunoflorescence stain for neutrophils in Figure 12 demonstrates the marked difference in numbers of infiltrating cells between the two extracts.

Interestingly, LAL testing of $B$. malayi and $A$. viteae extracts revealed $68.2 \mathrm{EU} / \mathrm{ml}$ and 3.79 EU/ml respectively. Unfortunately, a repeat $L A L$ test was not possible to perform due to shortage of extract. Again, with the recent insight into Wolbachia's genome rendering the existence of Wolbachia-LPS unlikely [40, 80], the above results have to be interpreted with some precaution as discussed in detail later. Briefly, the higher endotoxin level may explain the increased number of neutrophils seen with the $B$. malayi extract compared to the untreated OvAg extract in the previous experiment, and it could also account for the difference in inflammatory response evocated by the $B$. malayi and $A$. viteae extracts. The fact that speaks for Wolbachia rather than endotoxin as the important piece in this pathogenesis puzzle is that the Wolbachia-free $A$. viteae reagent overall produces only a minor inflammatory response despite its endotoxin level being similar to the untreated OvAg extract which caused significant pathology. On the other hand, differences in filarial antigens with different potencies to cause disease can also be confounding factors in interpretation of these results.
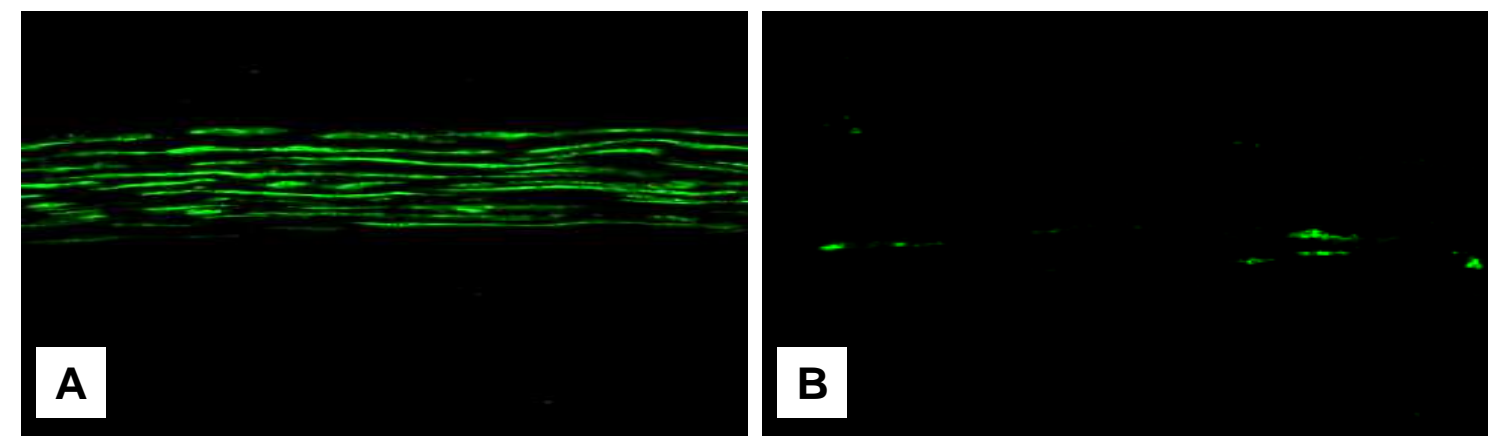

Figure 12 Increased neutrophil recruitment to the cornea with Wolbachia containing $B$. malayi extract (A) compared to endosymbiont free $\boldsymbol{A}$. viteae antigen (B). Neutrophils stained with anti-neutrophil antibody NIMP-R/14 and visualized by fluorescence microscopy (original magnification is $\times 600)$. Images by A. von Saint André. 


\section{Toll - like receptor 4 dependence of 0 . volvulus keratitis}

To determine if Wolbachia mediate corneal pathology by activating Toll-like receptor (TLR) 4, untreated $O$. volvulus extracts (i.e. containing Wolbachia) were injected into corneas of $\mathrm{C} 3 \mathrm{H} / \mathrm{HeJ}$ mice, which do not harbor an intact TLR4 and are hyporesponsive to LPS due to a single missense mutation within the TLR4 coding sequence [74]. The inflammatory response was compared with congenic, TLR4-mutant $\mathrm{C} 3 \mathrm{H} / \mathrm{HeN}$ mice. Figure 13 shows that in $\mathrm{C} 3 \mathrm{H} / \mathrm{HeJ}$ mice stromal

Stromal Thickness $(\mu \mathrm{m})$

\section{Stromal Haze ( $\mu \mathrm{m} \times$ intensity)}
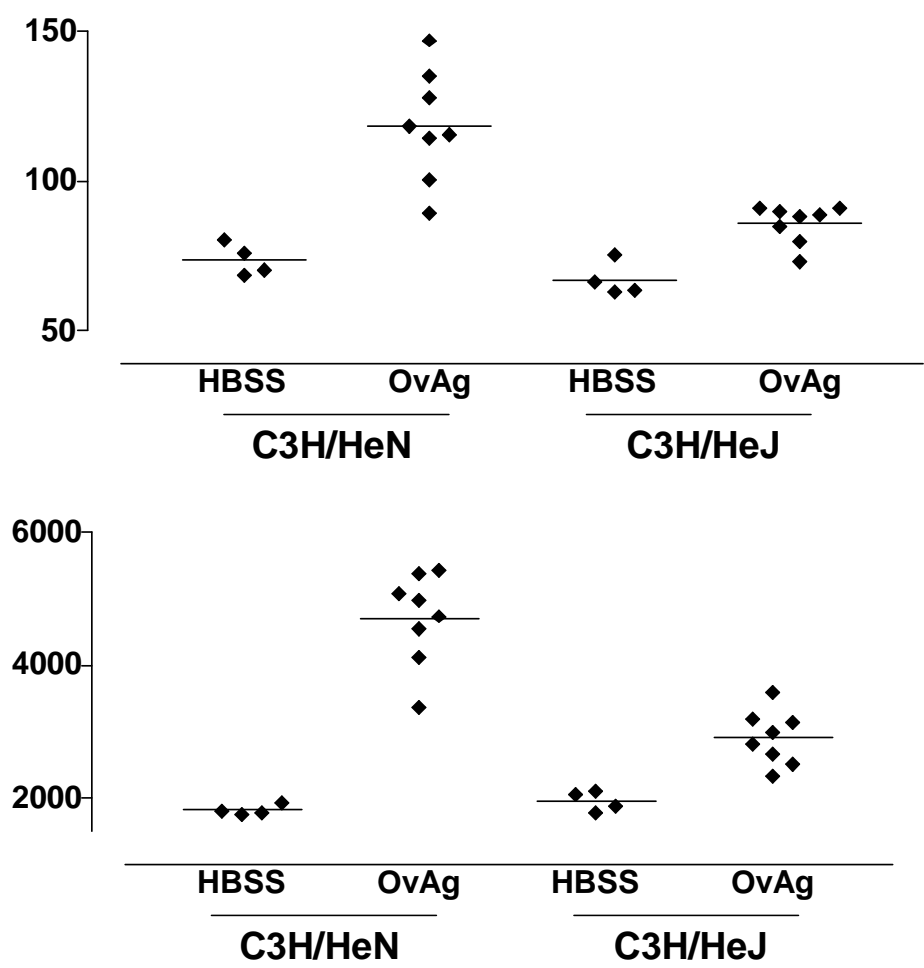

Neutrophils (per $5 \mu \mathrm{m}$ section)

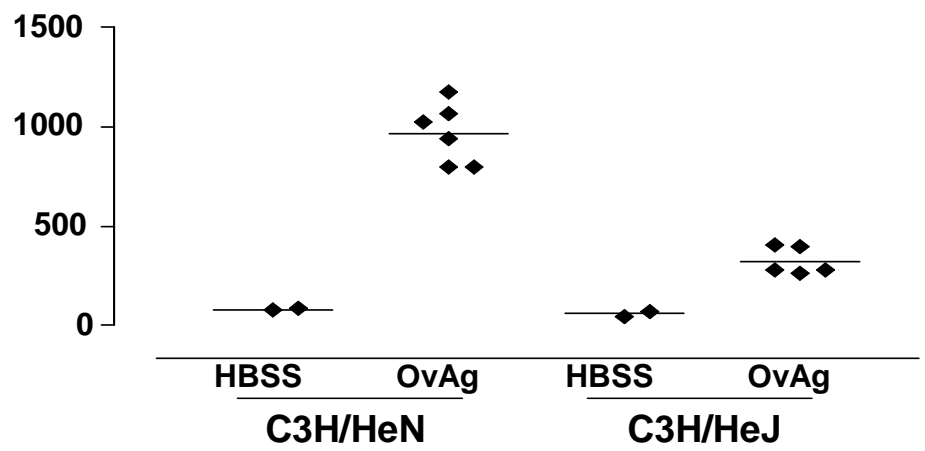

Figure 13 Decreased stromal thickness, stromal haze and neutrophil recruitment are found in TLR4-mutant $\mathrm{C} 3 \mathrm{H} / \mathrm{HeJ}$ mice challenged with Wolbachia-containing $O$. volvulus antigen. In contrary, TLR4-intact $\mathrm{C} 3 \mathrm{H} / \mathrm{HeN}$ mice show a significantly higher inflammatory response. Data points represent individual corneas from a single experiment. 
thickness, stromal haze and neutrophil recruitment were significantly diminished compared with wild-type mice $(p=0.0003$ for stromal thickness; $p<0.0001$ for stromal haze; $p<0.0001$ for neutrophil recruitment), indicating that TLR4 participates in regulating the development of $O$. volvulus keratitis. Two repeat experiments led to comparable results. This finding proposes an important role of Wolbachia bacteria or bacterial products in the pathogenic process of river blindness. LAL testing of this OvAg extract was $3.83 \mathrm{EU} / \mathrm{ml}$, again raising the question of endotoxin contamination explaining part of these results.

\section{TLR4 and Wolbachia dependent expression of PECAM-1 and of neutrophil chemokines MIP-2 and KC}

Since the cornea is an avascular tissue, cellular infiltration originates from limbal vessels in the peripheral region of the cornea, and progresses toward the central cornea where the initial stimulus (injection of parasite antigens) is induced. This experiment identifies whether essential mediators of neutrophil recruitment are dependent on the presence of Wolbachia. Expression of Platelet Endothelial Cell Adhesion molecule (PECAM) -1, Macrophage Inflammatory Protein (MIP)-2 and $K C$ are all essential for neutrophil recruitment to the cornea [52, 53, 58]. As Figure 14 demonstrates, their expression is significantly diminished in TLR4-mutant $\mathrm{C} 3 \mathrm{H} / \mathrm{HeJ}$ mice compared with wild-type mice $(\mathrm{p}=0.0054$ for PECAM-1; $\mathrm{p}=$ 0.0007 for MIP-2; $p<0.0001$ for $\mathrm{KC}$ ). Both mouse strains were challenged with intrastromal injections of untreated and therefore endosymbiont-containing $O$. volvulus antigen 24 hours prior to sacrificing the animals. Figure 15 shows the typical appearance of limbal vessels stained immunohistochemically for PECAM1 , with an obviously elevated expression of this adhesion molecule in wild-type mice. These findings which were confirmed by two repeat experiments indicate a regulatory role of TLR4 in O. volvulus keratitis by modulating the expression of PECAM-1, MIP-2 and KC in the cornea. Since the OvAg extract used here had an LAL level of $3.83 \mathrm{EU} / \mathrm{ml}$, the same considerations of an endotoxin related inflammatory effect apply in interpreting the above results.

Interestingly, TNF- $\alpha$ which is also essential for leukocyte recruitment, induces KC and MIP-2 expression [58], and had been described as a key proinflammatory 


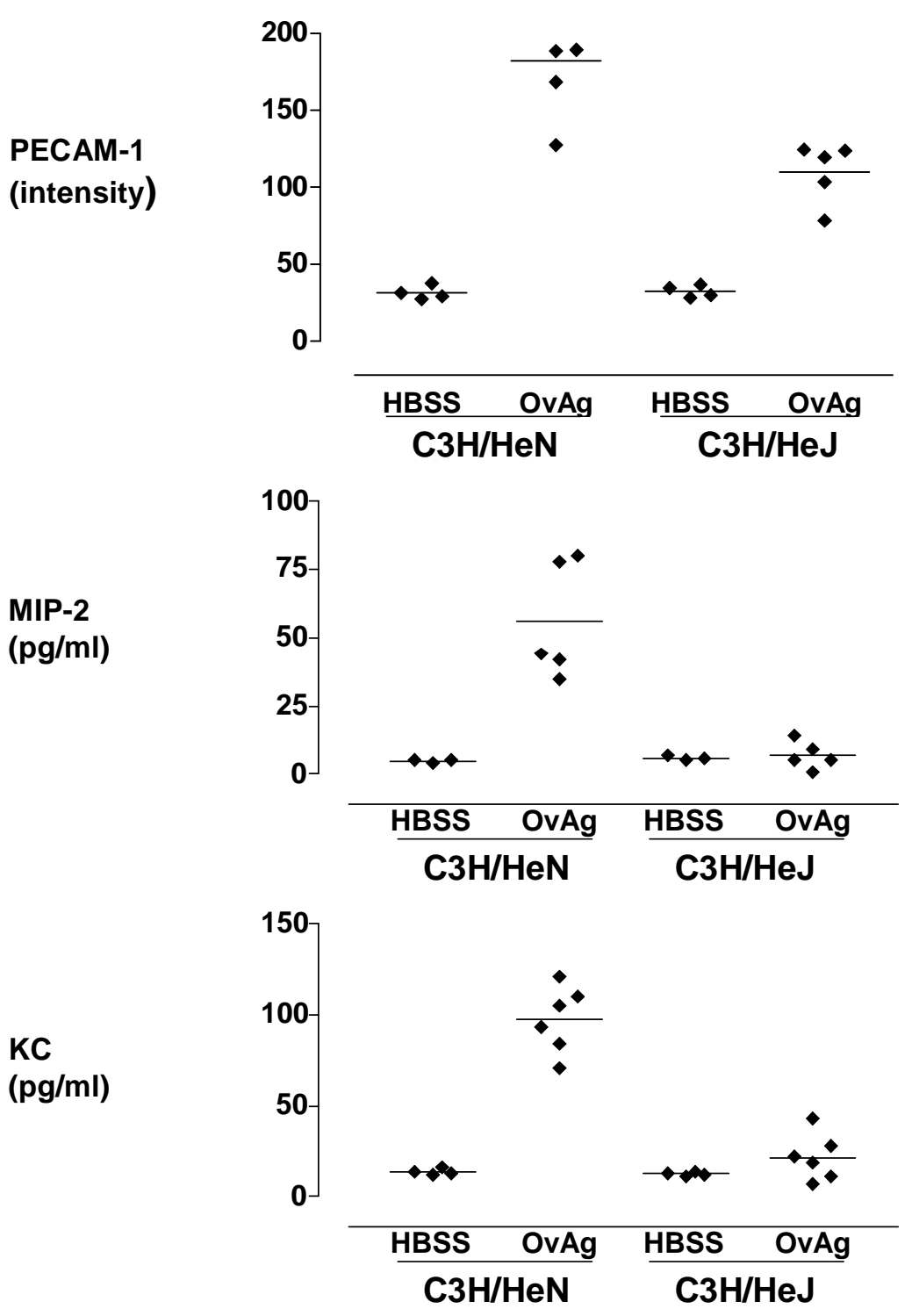

Figure 14 Decreased PECAM-1 expression and decreased MIP-2 and KC production in TLR4-mutant $\mathrm{C} 3 \mathrm{H} / \mathrm{HeJ}$ mice challenged with Wolbachia-containing 0 . volvulus antigen. In contrast, wild-type $\mathrm{C} 3 \mathrm{H} / \mathrm{HeN}$ mice show upregulation of these proinflammatory parameters in response to the same extract. Data points represent chemokine levels from individual corneas from a single experiment.

cytokine in filarial disease $[60,81]$ was undetectable in the corneal stroma of both $\mathrm{C} 3 \mathrm{H} / \mathrm{HeN}$ and $\mathrm{C} 3 \mathrm{H} / \mathrm{HeJ}$ mice (data not shown). This may have been caused by technical errors. However, this finding may also be due to a non-representative time point chosen for the detection of TNF- $\alpha$ in this experiment, since all cytokines were measured at 24 hours after intracorneal injection of parasite extract. Brattig et al found significant TNF- $\alpha$ production after 4 hours of stimulating human monocyte cultures with OvAg of untreated patients and decreasing levels after 24 hours [61]. Gillette-Ferguson et al [63] on the other hand were able to measure 

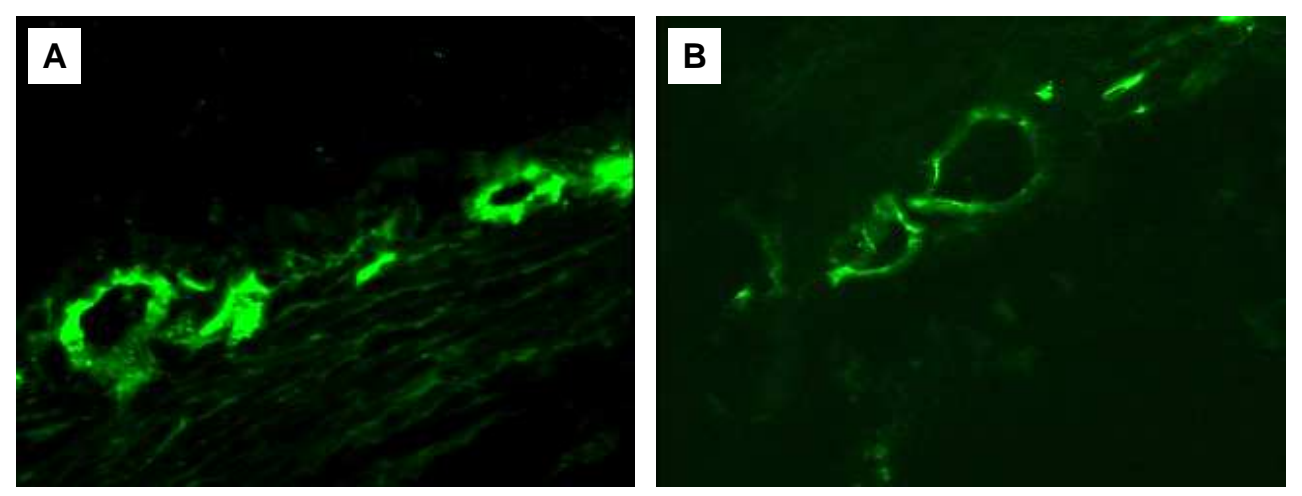

Figure 15 Increased staining intensity for PECAM-1 in TLR4-responsive $\mathrm{C} 3 \mathrm{H} / \mathrm{HeN}$ mice (A) compared to TLR4-mutant $\mathbf{C} 3 \mathrm{H} / \mathrm{HeJ}$ mice (B). Mice were sacrificed 24 hours after intracorneal injection of untreated $O$. volvulus antigen; $5 \mu \mathrm{m}$ sections of murine eyes were immunostained with mouse antibody against PECAM-1 and visualized using FITC anti-rat IgG. Original magnification $x$ 400. Images by A. von Saint André.

TNF- $\alpha$ levels in a dose dependent manner after 18 hours of incubation of murine neutrophil populations stimulated with Wolbachia-containing parasite extract.

In accordance to the TLR4 dependence of PECAM-1 and KC upregulation, their expression was diminished in corneas of C57BL/6 mice injected with OvAg/doxy (not containing Wolbachia) compared to those challenged with untreated Wolbachia-containing extract ( $p<0.0001$ for PECAM-1; $p=0.0313$ for KC) (Figure 16). These results which were reproduced twice, point again to the role of Wolbachia in recruitment of neutrophils to the cornea and thereby developing keratitis. 

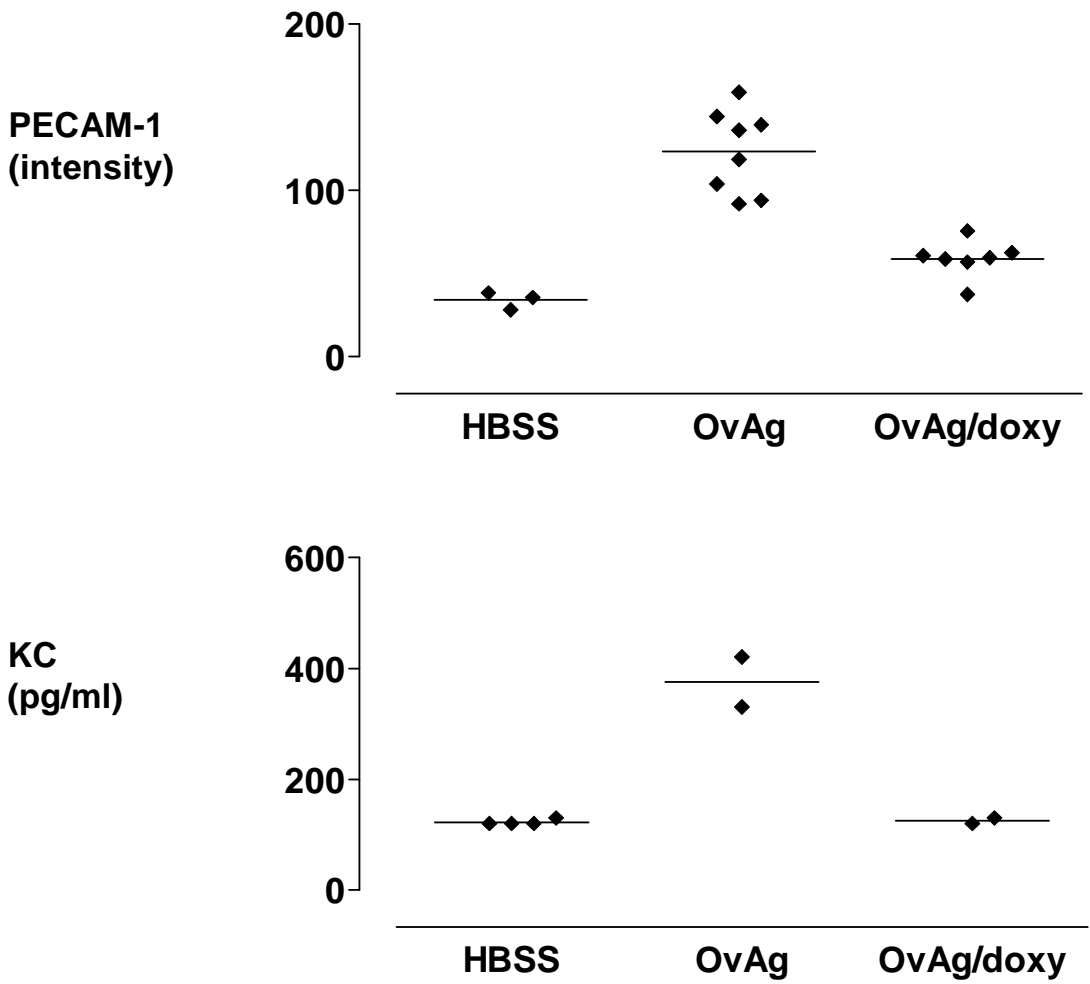

Figure 16 Reduced PECAM-1 expression and KC production with doxycycline treated $O$. volvulus extract in C57BL/6 mice. In contrast, untreated Wolbachia-containing extract revealed marked upregulation of the adhesion molecule PECAM-1 and the neutrophil chemokine KC. Data points represent chemokine levels from individual corneas from a single experiment. 


\section{DISCUSSION}

\section{Wolbachia as inducer of pathology}

This study demonstrates in a mouse model of onchocerca keratitis that development of corneal opacification is associated with the presence of Wolbachia endobacteria, neutrophils and the innate immune system. This was a surprising finding, since until then abundant literature suggested that filarial and other helminth infections are regulated by Th2 cell- and eosinophil responses [77, 82-85]. Three different approaches were used to demonstrate Wolbachia's important role in corneal pathology:

Firstly, Wolbachia-depleted extracts from doxycycline treated onchocerciasis patients led to a diminished inflammatory response in murine corneas compared to untreated, i.e. Wolbachia containing antigen. The decreased cell recruitment observed with doxycycline treated extracts involved neutrophils, but not eosinophils. This finding demonstrated that the presence of Wolbachia increases neutrophil recruitment. Furthermore, these results made clear that the number of neutrophils is a determining factor for the degree of pathology, since Wolbachiadepleted extracts led to less neutrophil infiltrate with less stromal thickness and haze. This conclusion was consistent with previous observations in which inhibiting recruitment of neutrophils, but not eosinophils to the corneal stroma resulted in significantly reduced corneal disease [52, 53]. Similarly, experiments using IL-5 knock-out mice which do not produce any eosinophils showed a sustained neutrophil infiltrate together with an exacerbated keratitis [64]. The difference in inflammatory response evoked by the treated versus untreated $O$. volvulus extract is likely due to the Wolbachia compound, although the presence of endotoxin in both reagents might contribute to some and at this point not known degree of neutrophil recruitment to the cornea simply due to this contamination. At the time these experiments were performed, it was thought that Wolbachia contained LPS which was responsible for the observed Wolbachia related immune response and would explain both the decreased endotoxin level and pathology seen with doxycycline treated antigen. As mentioned earlier, genomic sequencing has, however, shown that Wolbachia lack the genes required for LPS biosynthesis $[40,80]$. In the mean time, the search for the molecular nature of 
Wolbachia's stimulatory activity has focused on other candidate molecules found on the surface membrane of the bacteria. Wolbachia surface protein (WSP) has been shown in multiple experiments to strongly activate the innate immune system via TLR (see below) and could well explain not only the above, but also the findings of the second step in this study:

Extracts from Wolbachia-containing B. malayi revealed markedly more pathology than endosymbiont-free $A$. viteae antigen. This again pointed at the role of Wolbachia in development of disease. The relatively high endotoxin content of the B. malayi extract is possibly part of the reason why this reagent attracted far more neutrophils than its Wolbachia containing OvAg counterpart in the prior experiment. The $A$. viteae extract was also found to have an endotoxin level almost as high as the untreated $O$. volulus antigen. Interestingly, despite the similar endotoxin level, the inflammatory reaction caused by Wolbachia-free $A$. viteae was very mild compared to the Wolbachia containing OvAg. This observation points to Wolbachia as inducer of pathology, rather than contaminant LPS. Another uncertainty in interpreting the above results is to what extent the filarial proteins derived from different helminthes contribute to these findings, although the same protein concentration was used for all experiments.

Thirdly, TLR4 dependence was shown to exist for the inflammatory response to Wolbachia harboring $O$. volvulus antigen. TLRs are a family of at least 10 transmembrane proteins that are highly conserved in vertebrates and invertebrates, and represent a first line of defense by detecting conserved pathogen-associated molecular patterns (PAMPs) [86]. Cell stimulation through TLRs triggers activation of the transcription factor NF-KB that results in activation of genes encoding proinflammatory cytokines such as TNF- $\alpha$ [87] and II-8 or the murine homologue KC [73]. TLR4 is one of the most important TLRs that mediates signals for a broad spectrum of ligands including fatty acids, oligosaccharides, LPS and heat shock proteins. Mice deficient in functional TLR4 therefore produced only very mild inflammatory responses despite the presence of Wolbachia. Although this experiment was done with O. volvulus antigen containing a small amount of endotoxin and therefore the potential of confounding these results, Brattig et al showed that recombinant WSP activates TLR4 and TLR2 [62]. Several other reports also indicate that Wolbachia stimulates innate immune responses via TLR: Wolbachia activation of macrophages is decreased in 
TLR4 - mutant $\mathrm{C} 3 \mathrm{H} / \mathrm{HeJ}$ mice [60]; mice deficient in myeloid differentiation factor 88 (MyD88), which is common to the signaling pathways of TLR2 and TLR4, do not develop keratitis in response to $O$. volvulus antigens or to isolated Wolbachia bacteria [88].

Looking further into Wolbachia mediated mechanisms of neutrophil recruitment to the cornea, this study showed that expression of the adhesion molecule PECAM1 which mediates leukocyte passage across endothelial cells and basement membranes [89, 90] was dependent on the presence of functional TLR4 and Wolbachia respectively. TLR4 - mutant mice challenged with untreated OvAg, as well as C57BL/6 mice exposed to OvAg/doxy extracts demonstrated decreased PECAM-1 expression and therefore a decreased number of neutrophils with less pathology. As neutrophils also express PECAM-1, it is likely that homophilic interactions between PECAM-1 on leukocytes and PECAM-1 on endothelial cells $[89,91-93]$ are involved in neutrophil recruitment to the cornea.

Upregulation of the CXC chemokines KC and MIP-2 - essential mediators for neutrophil recruitment in mice and II-8 functional homologues - was also found to be dependent on the presence of functional TLR4 and Wolbachia. This observation is consistent with a study by Gillette-Ferguson's et al [63], in which isolated Wolbachia organisms stimulated in vitro production of MIP-2 and KC by neutrophils in a dose-dependent manner. Similarly, these cytokines were induced by filarial extracts containing Wolbachia organisms, but not by Wolbachiadepleted extracts.

In summary, the results of this study indicate that the innate immune system and Wolbachia endobacteria play an important role in the inflammatory response associated with the pathogenesis of onchocerca keratitis which is probably consistent with the following sequence of events: microfilariae invade the cornea, where they eventually die and release Wolbachia endobacteria into the confined environment of the corneal stroma. Wolbachia products activate the innate inflammatory response through TLR4 and according to Gillette-Ferguson et al also through TLR2 on resident corneal epithelial cells and keratocytes. Activated keratocytes can mature into stromal fibroblasts which produce proinflammatory and CXC chemokines [66]. TLR4 and TLR2 activation also results in PECAM-1 expression on peripheral limbal vessels, facilitating recruitment of neutrophils to 
the avascular corneal stroma. As shown in a recent study [63], infiltrating neutrophils in the cornea surround the microfilariae and ingest Wolbachia organisms. As neutrophils express functional TLR2 and TLR4, they can produce MIP-2 and KC in response to Wolbachia, which mediates further neutrophil infiltration, degranulation and release of cytotoxic products such as nitric oxide, oxygen free radicals, and matrix metalloproteinase. The cytotoxic effect on keratocytes and corneal endothelial cells, the cells responsible for maintaining corneal clarity, will lead to increased stromal thickness and haze, resulting in visual impairment and eventually blindness (Figure 17).

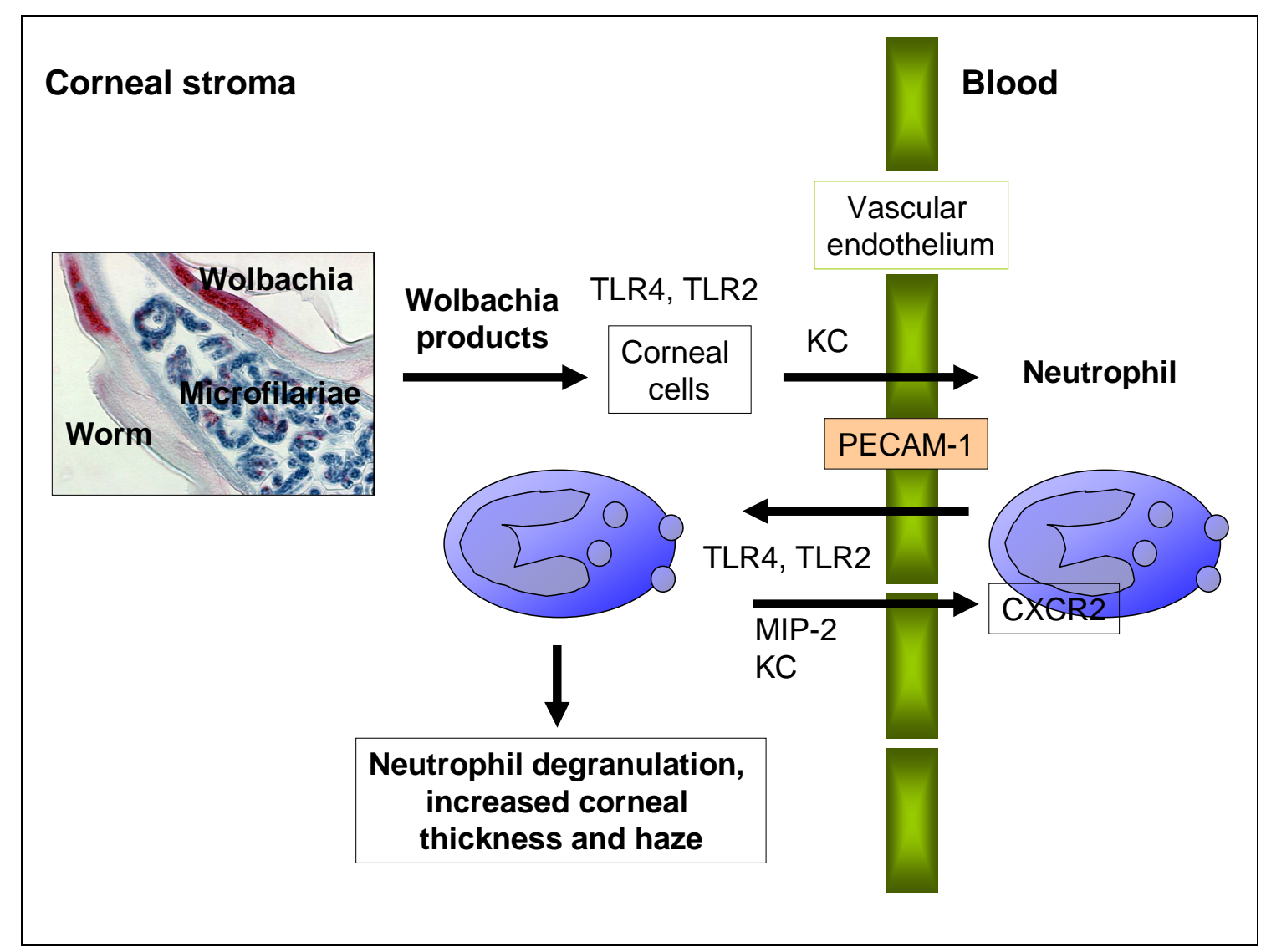

Figure 17 Proposed sequence of events of innate immune responses in onchocerca keratitis. See text for detailed explanation. Image by A. von Saint André.

To determine if TLR4 also regulates keratitis in the presence of an adaptive immune response, in a follow-up experiment (data not shown) $\mathrm{C} 3 \mathrm{H} / \mathrm{HeN}$ and $\mathrm{C} 3 \mathrm{H} / \mathrm{HeJ}$ were immunized with 3 weekly subcutaneous injections of untreated OvAg extract as a model for chronic infection [94]. Corneas were then injected intrastromally with the same Wolbachia-containing extract. Stromal thickness, 
stromal haze, and neutrophil infiltration were assessed at 24 to 72 hours, and eosinophil infiltration was examined after 72 hours, when there is a pronounced eosinophil infiltration in immunocompetent mice $[50,52]$. TLR4-mutant $\mathrm{C} 3 \mathrm{H} / \mathrm{HeJ}$ mice had significantly less stromal thickness, stromal haze, and numbers of neutrophils than wild-type $\mathrm{C} 3 \mathrm{H} / \mathrm{HeN}$ mice. However, there was no significant difference in eosinophil numbers between $\mathrm{C} 3 \mathrm{H} / \mathrm{HeJ}$ and $\mathrm{C} 3 \mathrm{H} / \mathrm{HeN}$ mice at 72 hours, indicating that in contrast to neutrophils, eosinophil recruitment to the cornea is not regulated by TLR4. When taken together, these findings demonstrate an essential role for Wolbachia and TLR4 in corneal pathology, even in the presence of an adaptive immune response.

Multiple other studies support an important role for Wolbachia and the innate response in humans and animals infected with onchocerciasis and lymphatic filariasis. Recombinant, purified Wolbachia surface protein (WSP), an abundantly expressed and highly conserved protein in filarial Wolbachia, acts as an inducer of the innate human immune system through both TLR2 and TLR4 dependent pathways [62, 95]. Interestingly, WSP was also reported to induce IL-8 production by neutrophils, consistent with an upregulation of its murine equivalents MIP-2 and $\mathrm{KC}$ in this study.

Wolbachia are released into the blood following anti-filarial chemotherapy of onchocerciasis, with peak DNA levels correlating with clinical reaction scores [96]. This so-called Mazzotti reaction is characterized by an acute papular onchodermatitis, lymphadenitis, pruritus, rash as well as fever, and sometimes, hypotension that may exacerbate to shock. In the early phase of the Mazzotti reaction a burst of circulating antibacterial acute phase reactants such as TNF- $\alpha$ and II-6 as well as transient neutrophilia coincide with the above pathological manifestations [38, 77, 96, 97]. Interestingly, locally applied DEC to the skin of infected individuals causes abscess formation that is associated with an intense neutrophil rather than eosinophil infiltrate [98] - likely due to the release of endobacterial products. Wolbachia are also responsible for the recruitment and activation of neutrophils in the granulomatous response: Neutrophils infiltrate adult worm subcutaneous nodules in the presence of the endosymbiont, and disappear when Wolbachia are cleared using doxycycline [81, 99]. Wolbachia-induced cell activation may also result in the abundant binding of defensin, a major neutrophil 
constituent, to the surface of adult female O. volvulus worms [100]. In addition, neutrophils are thought to be involved in the generation of a cyst at the anterior end of female worms, apparently as an aid for uptake of host nutrients and for facilitating mating with males. Thus, neutrophils recruited by endobacterial chemoattractants may contribute to the survival of the parasite [100, 101]. In tetracycline-treated patients, no accumulations of neutrophils in the vicinity of filariae are observed, and $O$. volvulus extracts from these patients show reduced chemotactic activity [102].

Similarly, in an animal model with the red deer (C. elaphus) which is often the host of several onchocerca species in the same animal, massive neutrophil infiltration was observed around the endobacteria-positive deer filaria O. jakutensis, while it was absent around the endobacteria-free deer filaria O. flexuosa. Since onchocercomas of both species were studied from the same animal, variation of host reactivity in different deer can be excluded as a cause for the difference in neutrophil chemotaxis [81].

A recent study comparing the different "forest" and "savanna" strains of $O$. volvulus of West Africa found to have a significantly greater ratio of Wolbachia DNA to nuclear DNA in the severe, ocular disease causing "savanna" strain, supporting the role of bacteria in the pathogenesis of ocular onchocerciasis [103]. In summary, all the above studies suggest that $O$. volvulus worms may release Wolbachia or Wolbachia products and thereby promote innate inflammatory responses both adjacent to the worm and systemically.

In lymphatic filariasis, similarly to the in vivo experiments in this study, soluble extracts of $B$. malayi adults or microfilariae also induced a potent innate inflammatory response in vitro $[60,63]$. The activation of inflammation requires CD14 and TLR4 pattern recognition receptors and the activity is lost after antibiotic depletion of bacteria and is absent from soluble extracts derived from aposymbiotic species (A. viteae and L. Loa) [60, 104]. Similar to the Mazzotti reaction described above for onchocerciasis, inflammatory reactions also occur following anti-lymphatic filariasis drug treatment particularily in patients with high parasite burdens. Severe adverse reactions are associated with the increase in systemic proinflammatory cytokines and inflammatory mediators [97]. PCR and immunoelectron microscopy analysis of plasma samples following the treatment 
of $B$. malayi with DEC showed the persistent presence of Wolbachia in patients with severe systemic inflammation [38]. Recently, clinical trials have provided further evidence to support the role of Wolbachia in the presentation of adverse reactions: In patients infected with $W$. bancrofti, prior treatment with a three week course of doxycycline to deplete Wolbachia prevented moderate adverse reactions to albendazole and ivermectin, whereas for individuals in the placebo group plasma Wolbachia levels were related to the incidence of adverse reactions, as well as the levels of pro-inflammatory cytokines and the pretreatment microfilarial load [105].

In animal models of lymphatic filariasis, the production of TNF- $\alpha$ following the chemotherapy of $B$. malayi microfilariae only occurred in mice with an intact TLR4, suggesting that the release of Wolbachia is responsible for this inflammation [60]. Further effects of TLR4 mediated responses have been reported in mice infected with Wolbachia harboring Litomosoides sigmodontis [106]: In $\mathrm{C} 3 \mathrm{H} / \mathrm{HeN}$ mice infection results in adult female development including females containing mature microfilariae, but no detection of free microfilariae. Infection of TLR4 mutant $\mathrm{C} 3 \mathrm{H} / \mathrm{HeJ}$ mice produced worms with an increased fertility and the production of microfilariae, suggesting TLR4 mediated immune regulation of worm fertility.

Taken together, over the past several years there has been a considerable increase in the knowledge of the biological significance of Wolbachia for their filarial hosts, and the results of this and the above mentioned studies changed our understanding of the immunopathology of filariasis.

\section{Wolbachia endobacteria - new targets for therapy}

Onchocerciasis is the second most common infectious blinding disease in the developing world, responsible for an estimated 500,000 people with severe visual impairment and another 270,000 with blindness worldwide. A WHO Expert Committee in 1995 [1] estimated that over 120 million people are at risk with some 17.7 million infected, $99 \%$ of whom live in Africa. Onchocerciasis is a disease of remote, rural, poor populations. People have in the past abandoned fertile land along the rivers that harbor the breeding sites of the Simulium, for fear of going blind. While the situation has improved due to the above mentioned control 
programs in the savannah of West Africa [107], people with unsightly skin lesions still suffer major social and economical consequences [108] and according to a recent epidemiology study, approximately $5 \%$ of deaths in endemic areas of West Africa can be attributed to the effects of onchocerciasis [109].

The general idea in mass chemotherapy is to abolish microfilariae in skin and blood, in a large enough proportion of the population so that the cycle of transmission (see Figure 2) is inhibited. Using current tools, onchocerciasis appears to be ineradicable in Africa by both vector control and chemotherapy [7]: Ivermectin, a macrocyclic lactone [110] acts through inhibition of glutamate-gated chloride channels of microfilariae, effectively immobilizing them. It rapidly reduces the number of skin microfilariae, but depletes them only for a few months, after which they reappear at amounts of $20 \%$ or more of pretreatment levels within a year [111]. This seems to be sufficient for transmission to continue [112]. Ivermectin does not kill the long lived adult worms, nor does is permanently stop microfilarial production since its embryocital activity seems to be mainly restricted to the late stages of microfilarial development, leaving early embryogenesis intact [111]. Repeated rounds of treatment reduce fecundity of female worms [113], but this effect is not complete and given the longevity of the adult worm ( $>14$ years), interruption of mass treatment or too low coverage in the population, can quickly lead to reemergence of onchocerciasis [8].

Although the lack of ivermectin resistance is so far obviously encouraging, it is hardly surprising considering also the lack of any comprehensive monitoring strategies. The use of a single drug and the need of sustained treatment for decades have raised concerns over the potential development of resistance in onchocerciasis which were strongly endorsed at the Conference on Eradicability of Onchocerciasis in January 2002 [7]. Despite the lack of formal evidence for drug resistance, several cases of 'non or poor responsiveness' to treatment of onchocerciasis with ivermectin have been reported [7, 114]. Most recent observations suggest the development of resistant adult parasite populations in Ghana [10]. Also genetic selection of $O$. volvulus by ivermectin has been reported [9, 115-120] and drug resistance to ivermectin has already become a major obstacle to the control of nematode parasites of lifestock worldwide [121]. The need for new drugs to achieve elimination of transmission and hence elimination of onchocerciasis as a public health problem is undisputed. A list of drugs exists 
[122-124] which show effects against microfilariae or embryos and thus might be developed for combination with Ivermectin or selected use. However, as reviewed by Awazi [125] development has been stopped since the effects are either inferior to Ivermectin or do not improve its efficacy. Similar to onchocerciasis, in lymphatic filariasis concerns regarding drug resistance exist not only for Ivermectin, but also for the other agents that are the mainstay of treatment, DEC and Albendazole [126].

Because of the essential role for the worms' reproduction and therefore their role as obligatory symbionts in filarial nematodes, Wolbachia have emerged as the only target for chemotherapy that results in long-term sterility of the worms in human onchocerciasis $[127,128]$, a priority research objective for the WHO for the last 20 years [1]. The fact that doxycycline is already registered allowed for a quick transition from animal to phase lla studies on human onchocerciasis. Initial trials using a six week course of doxycycline led to depletion of Wolbachia and resulted in a block of embryogenesis in adult worms, which persisted for up to 2 years after start of treatment [31, 127, 129]. In contrast, patients treated with ivermectin alone showed an increase in microfilarial counts as early as four months after administration of ivermectin. The apparent permanent block in embryogenesis after doxycycline treatment was reflected in sustained reduction in skin microfilariae. A limited but significant macrofilaricidal effect was also observed, which had been reported earlier in bovine onchocerciasis [33]. Depletion of Wolbachia and sustained amicrofilaremia for almost 2 years after doxycycline treatment was also demonstrated in human lymphatic filariasis patients infected with $W$. bangrofti [130]. A recent placebo-controlled trial in humans infected with $W$. bangrofti has shown a clear macrofilaricidal effect of doxycycline when administered for 8 weeks at $200 \mathrm{mg} / \mathrm{day}$, associated with $95 \%$ reduction of Wolbachia levels [131]. The results from human trials thus far have unequivocally demonstrated the superior pharmacological efficacy of doxycycline for both onchocerciasis and lymphatic filariasis. The long period of treatment (six weeks or more) and the known contraindications to doxycycline (pregnant or breastfeeding women, children up to 9 years of age) preclude its application for mass treatment at the moment. Further research is needed to exploit the principle of targeting Wolbachia in several ways, using other already existing antibiotics, 
combinations and designing new drugs on the basis of the information provided by the Wolbachia and filarial genome data.

So far, as agreed at the conferences in Hamburg and Atlanta [7, 132], individual treatment of people with imported infection or those leaving an endemic area for a long time are indications for doxycycline (100 mg/ day for six weeks). Another indication could be in formerly endemic areas where a high level of control has already been achieved and re-emergence should be prevented. Wolbachiatargeting antibiotics may also improve compliance with mass treatment programs by reducing side-effects due to the release of intact bacteria and Wolbachia products from dying nematodes [38]. Pretreatment targeting Wolbachia may abrogate inflammatory responses and allow a wider range of antifilarial drugs to be used - not only for onchocerciasis, but also for other filarial diseases.

In conclusion, the discovery of endosymbiotic bacteria infecting most species of filarial nematodes that are pathogenic to humans has opened exciting new avenues of research into the pathogenesis and immunology of filarial infections. In fact, the results of this study suggest a complete alteration in our understanding of the immunopathology of onchocerciasis. It is not simply the worm, but the Wolbachia endosymbiont that is a major contributor to the development of ocular onchocerciasis. Wolbachia therefore seems to have a double role as a new target for therapy: Firstly, clearance of Wolbachia by antibiotic treatment may reduce and prevent ocular onchocerciasis, since Wolbachia plays an essential role in the pathogenesis of this disease. Secondly, targeting Wolbachia leads to long-term sterilizing and possibly macrofilaricidal effects that have the potential to eliminate onchocerciasis as a public health problem. 


\section{SUMMARY}

Introduction: This study investigates the role of Wolbachia bacteria in the pathogenesis of $O$. volvulus keratitis in a mouse model. Wolbachia bacteria are essential symbionts of most filarial nematodes of importance for mankind.

Methods: Using a mouse model for river blindness in which soluble extracts of filarial nematodes are injected in the corneal stroma, changes in stromal thickness and haze of the cornea are observed by in vivo confocal microscopy, followed by immunohistochemical staining for neutrophils and PECAM-1, as well as ELISA of corneal chemokines. Reactions to filarial extracts containing Wolbachia are compared to those without the endosymbiont.

Results: The approach of characterizing Wolbachia's role in river blindness in this study is threefold. Firstly, Wolbachia-depleted extracts from doxycycline treated onchocerciasis patients led to a diminished inflammatory response in corneas of C57BL/6 mice compared to untreated, i.e. Wolbachia containing antigen. The decreased cell recruitment observed with doxycycline treated extracts involved neutrophils, but not eosinophils. This finding demonstrated that the presence of Wolbachia increases neutrophil recruitment. Secondly, extracts from Wolbachiacontaining $B$. malayi revealed markedly more pathology than endosymbiont-free A. viteae antigen. This again pointed at the role of Wolbachia in development of disease. Thirdly, Toll-like Receptor 4 (TLR4) dependence was shown to exist for the inflammatory response to Wolbachia harboring $O$. volvulus antigen by looking at the corneal pathology in TLR4-mutant $\mathrm{C} 3 \mathrm{H} / \mathrm{HeJ}$ mice, compared to the wildtype $\mathrm{C} 3 \mathrm{H} / \mathrm{HeN}$ strain. Investigating further Wolbachia mediated mechanisms of neutrophil recruitment to the cornea, this study also showed that expression of the adhesion molecule PECAM-1 in limbal vessels, as well as upregulation of the CXC chemokines KC and MIP-2 were dependent on the presence of functional TLR4 and Wolbachia respectively.

Conclusions: This study indicates that the innate immune system and Wolbachia endobacteria play an important role in the inflammatory response associated with the pathogenesis of onchocerca keratitis, suggesting a complete alteration in our understanding of the immunopathology of filariasis. 


\section{REFERENCES}

1. Onchocerciasis and its control. Report of a WHO Expert Committee on Onchocerciasis Control. World Health Organ Tech Rep Ser, 1995. 852: p. 1-104.

2. Buttner, D.W. and P. Racz, Macro- and microfilariae in nodules from onchocerciasis patients in the Yemen Arab Republic. Tropenmed Parasitol, 1983. 34(2): p. 113-21.

3. Duke, B.O., The population dynamics of Onchocerca volvulus in the human host. Trop Med Parasitol, 1993. 44(2): p. 61-8.

4. Newland, H.S., et al., Ocular manifestations of onchocerciasis in a rain forest area of west Africa. Br J Ophthalmol, 1991. 75(3): p. 163-9.

5. Richards, F.O., Jr., et al., Control of onchocerciasis today: status and challenges. Trends Parasitol, 2001. 17(12): p. 558-63.

6. Winnen, M., et al., Can ivermectin mass treatments eliminate onchocerciasis in Africa? Bull World Health Organ, 2002. 80(5): p. 384-91.

7. Dadzie, Y., M. Neira, and D. Hopkins, Final report of the Conference on the eradicability of Onchocerciasis. Filaria J, 2003. 2(1): p. 2.

8. Plaisier, A.P., et al., Required duration of combined annual ivermectin treatment and vector control in the Onchocerciasis Control Programme in west Africa. Bull World Health Organ, 1997. 75(3): p. 237-45.

9. Awadzi, K., et al., An investigation of persistent microfilaridermias despite multiple treatments with ivermectin, in two onchocerciasis-endemic foci in Ghana. Ann Trop Med Parasitol, 2004. 98(3): p. 231-49.

10. Osei-Atweneboana, M.Y., et al., Prevalence and intensity of Onchocerca volvulus infection and efficacy of ivermectin in endemic communities in Ghana: a two-phase epidemiological study. Lancet, 2007. 369(9578): p. 2021-9.

11. McLaren, D.J., et al., Micro-organisms in filarial larvae (Nematoda). Trans R Soc Trop Med Hyg, 1975. 69(5-6): p. 509-14.

12. Kozek, W.J. and H.F. Marroquin, Intracytoplasmic bacteria in Onchocerca volvulus. Am J Trop Med Hyg, 1977. 26(4): p. 663-78. 
13. Vincent, A.L., L.R. Ash, and S.P. Frommes, The ultrastructure of adult Brugia malayi (Brug, 1927) (Nematoda: Filarioidea). J Parasitol, 1975. 61(3): p. 499-512.

14. Stouthamer, R., J.A. Breeuwer, and G.D. Hurst, Wolbachia pipientis: microbial manipulator of arthropod reproduction. Annu Rev Microbiol, 1999. 53: p. 71-102.

15. Werren, J.H., W. Zhang, and L.R. Guo, Evolution and phylogeny of Wolbachia: reproductive parasites of arthropods. Proc $\mathrm{R}$ Soc Lond B Biol Sci, 1995. 261(1360): p. 55-63.

16. Buttner, D.W., et al., Obligatory symbiotic Wolbachia endobacteria are absent from Loa loa. Filaria J, 2003. 2(1): p. 10.

17. McGarry, H.F., et al., Evidence against Wolbachia symbiosis in Loa loa. Filaria J, 2003. 2(1): p. 9.

18. Bandi, C., et al., Phylogeny of Wolbachia in filarial nematodes. Proc R Soc Lond B Biol Sci, 1998. 265(1413): p. 2407-13.

19. Taylor, M.J. and A. Hoerauf, Wolbachia bacteria of filarial nematodes. Parasitol Today, 1999. 15(11): p. 437-42.

20. Dumler, J.S., et al., Reorganization of genera in the families Rickettsiaceae and Anaplasmataceae in the order Rickettsiales: unification of some species of Ehrlichia with Anaplasma, Cowdria with Ehrlichia and Ehrlichia with Neorickettsia, descriptions of six new species combinations and designation of Ehrlichia equi and 'HGE agent' as subjective synonyms of Ehrlichia phagocytophila. Int J Syst Evol Microbiol, 2001. 51(Pt 6): p. 214565.

21. Sironi, M., et al., Molecular evidence for a close relative of the arthropod endosymbiont Wolbachia in a filarial worm. Mol Biochem Parasitol, 1995. 74(2): p. 223-7.

22. Bandi, C., A.J. Trees, and N.W. Brattig, Wolbachia in filarial nematodes: evolutionary aspects and implications for the pathogenesis and treatment of filarial diseases. Vet Parasitol, 2001. 98(1-3): p. 215-38.

23. Lo, N., et al., How many wolbachia supergroups exist? Mol Biol Evol, 2002. 19(3): p. 341-6.

24. Casiraghi, M., et al., dnaA gene sequences from Wolbachia pipientis support subdivision into supergroups and provide no evidence for 
recombination in the lineages infecting nematodes. Parassitologia, 2003. 45(1): p. 13-8.

25. McGarry, H.F., G.L. Egerton, and M.J. Taylor, Population dynamics of Wolbachia bacterial endosymbionts in Brugia malayi. Mol Biochem Parasitol, 2004. 135(1): p. 57-67.

26. Kramer, L.H., et al., Immunohistochemical/immunogold detection and distribution of the endosymbiont Wolbachia of Dirofilaria immitis and Brugia pahangi using a polyclonal antiserum raised against WSP (Wolbachia surface protein). Parasitol Res, 2003. 89(5): p. 381-6.

27. Taylor, M.J., et al., $16 S$ rDNA phylogeny and ultrastructural characterization of Wolbachia intracellular bacteria of the filarial nematodes Brugia malayi, B. pahangi, and Wuchereria bancrofti. Exp Parasitol, 1999. 91(4): p. 356-61.

28. Sacchi, L., et al., Does fertilization in the filarial nematode Dirofilaria immitis occur through endocytosis of spermatozoa? Parasitology, 2002. 124(Pt 1): p. 87-95.

29. Kozek, W.J., What is new in the Wolbachia/Dirofilaria interaction? Vet Parasitol, 2005. 133(2-3): p. 127-32.

30. Hoerauf, A., Targeting of wolbachia endobacteria in litomosoides sigmodontis: comparison of tetracyclines with chloramphenicol, macrolides and ciprofloxacin. Trop Med Int Health, 2000. 5(4): p. 275-9.

31. Hoerauf, A., et al., Endosymbiotic bacteria in worms as targets for a novel chemotherapy in filariasis. Lancet, 2000. 355(9211): p. 1242-3.

32. Townson, S., et al., Antibiotics and Wolbachia in filarial nematodes: antifilarial activity of rifampicin, oxytetracycline and chloramphenicol against Onchocerca gutturosa, Onchocerca lienalis and Brugia pahangi. Ann Trop Med Parasitol, 2000. 94(8): p. 801-16.

33. Langworthy, N.G., et al., Macrofilaricidal activity of tetracycline against the filarial nematode Onchocerca ochengi: elimination of Wolbachia precedes worm death and suggests a dependent relationship. Proc Biol Sci, 2000. 267(1448): p. 1063-9.

34. Rao, R.U., H. Moussa, and G.J. Weil, Brugia malayi: effects of antibacterial agents on larval viability and development in vitro. Exp Parasitol, 2002. 101(1): p. 77-81. 
35. Smith, H.L. and T.V. Rajan, Tetracycline inhibits development of the infective-stage larvae of filarial nematodes in vitro. Exp Parasitol, 2000. 95(4): p. 265-70.

36. Rajan, T.V., Relationship of anti-microbial activity of tetracyclines to their ability to block the L3 to L4 molt of the human filarial parasite Brugia malayi. Am J Trop Med Hyg, 2004. 71(1): p. 24-8.

37. Chirgwin, S.R., et al., Brugia pahangi and Wolbachia: the kinetics of bacteria elimination, worm viability, and host responses following tetracycline treatment. Exp Parasitol, 2003. 103(1-2): p. 16-26.

38. Cross, H.F., et al., Severe reactions to filarial chemotherapy and release of Wolbachia endosymbionts into blood. Lancet, 2001. 358(9296): p. 1873-5.

39. Dedeine, F., et al., Removing symbiotic Wolbachia bacteria specifically inhibits oogenesis in a parasitic wasp. Proc Natl Acad Sci U S A, 2001. 98(11): p. 6247-52.

40. Foster, J., et al., The Wolbachia genome of Brugia malayi: endosymbiont evolution within a human pathogenic nematode. PLoS Biol, 2005. 3(4): p. e121.

41. Fenn, K. and M. Blaxter, Wolbachia genomes: revealing the biology of parasitism and mutualism. Trends Parasitol, 2006. 22(2): p. 60-5.

42. Bandi, C., et al., Effects of tetracycline on the filarial worms Brugia pahangi and Dirofilaria immitis and their bacterial endosymbionts Wolbachia. Int $\mathrm{J}$ Parasitol, 1999. 29(2): p. 357-64.

43. Kenyon, K., Morphology and pathologic responses of the cornea to disease. in Smolin G. Thoft RA (eds): The cornea. Scientific Foundations and Clinical Practice. 1987, Boston: Little, Brown and Company. pp 63-99.

44. Pearlman, E., et al., Interleukin 4 and $T$ helper type 2 cells are required for development of experimental onchocercal keratitis (river blindness). J Exp Med, 1995. 182(4): p. 931-40.

45. Chakravarti, B., et al., Immune-mediated Onchocerca volvulus sclerosing keratitis in the mouse. Exp Eye Res, 1993. 57(1): p. 21-7.

46. Chakravarti, B., et al., Infiltration of CD4+ $T$ cells into cornea during development of Onchocerca volvulus-induced experimental sclerosing keratitis in mice. Cell Immunol, 1994. 159(2): p. 306-14. 
47. Chakravarti, B., et al., In vivo molecular analysis of cytokines in a murine model of ocular onchocerciasis. I. Up-regulation of IL-4 and IL-5 mRNAs and not IL-2 and IFN gamma mRNAs in the cornea due to experimental interstitial keratitis. Immunol Lett, 1996. 54(1): p. 59-64.

48. Hall, L.R. and E. Pearlman, Pathogenesis of onchocercal keratitis (River blindness). Clin Microbiol Rev, 1999. 12(3): p. 445-53.

49. Pearlman, E. and L.R. Hall, Immune mechanisms in Onchocerca volvulusmediated corneal disease (river blindness). Parasite Immunol, 2000. 22(12): p. 625-31.

50. Hall, L.R., E. Diaconu, and E. Pearlman, A dominant role for FC gamma receptors in antibody-dependent corneal inflammation. J Immunol, 2001. 167(2): p. 919-25.

51. Hall, L.R., et al., An essential role for antibody in neutrophil and eosinophil recruitment to the cornea: $B$ cell-deficient (microMT) mice fail to develop Th2-dependent, helminth-mediated keratitis. J Immunol, 1999. 163(9): p. 4970-5.

52. Kaifi, J.T., E. Diaconu, and E. Pearlman, Distinct roles for PECAM-1, ICAM-1, and VCAM-1 in recruitment of neutrophils and eosinophils to the cornea in ocular onchocerciasis (river blindness). J Immunol, 2001. 166(11): p. 6795-801.

53. Hall, L.R., et al., CXC chemokine receptor 2 but not $C$-C chemokine receptor 1 expression is essential for neutrophil recruitment to the cornea in helminth-mediated keratitis (river blindness). J Immunol, 2001. 166(6): p. 4035-41.

54. Rothenberg, M.E., et al., Targeted disruption of the chemokine eotaxin partially reduces antigen-induced tissue eosinophilia. J Exp Med, 1997. 185(4): p. 785-90.

55. Rollins, B.J., Chemokines. Blood, 1997. 90(3): p. 909-28.

56. Harada, A., et al., Essential involvement of interleukin-8 (IL-8) in acute inflammation. J Leukoc Biol, 1994. 56(5): p. 559-64.

57. Van Zee, K.J., et al., IL-8 in septic shock, endotoxemia, and after IL-1 administration. J Immunol, 1991. 146(10): p. 3478-82. 
58. Tessier, P.A., et al., Chemokine networks in vivo: involvement of $C-X-C$ and $C-C$ chemokines in neutrophil extravasation in vivo in response to TNF-alpha. J Immunol, 1997. 159(7): p. 3595-602.

59. Al-Qaoud, K.M., et al., A new mechanism for IL-5-dependent helminth control: neutrophil accumulation and neutrophil-mediated worm encapsulation in murine filariasis are abolished in the absence of IL-5. Int Immunol, 2000. 12(6): p. 899-908.

60. Taylor, M.J., H.F. Cross, and K. Bilo, Inflammatory responses induced by the filarial nematode Brugia malayi are mediated by lipopolysaccharide-like activity from endosymbiotic Wolbachia bacteria. J Exp Med, 2000. 191(8): p. 1429-36.

61. Brattig, N.W., et al., Lipopolysaccharide-like molecules derived from Wolbachia endobacteria of the filaria Onchocerca volvulus are candidate mediators in the sequence of inflammatory and antiinflammatory responses of human monocytes. Microbes Infect, 2000. 2(10): p. 1147-57.

62. Brattig, N.W., et al., The major surface protein of Wolbachia endosymbionts in filarial nematodes elicits immune responses through TLR2 and TLR4. J Immunol, 2004. 173(1): p. 437-45.

63. Gillette-Ferguson, I., et al., Wolbachia-induced neutrophil activation in a mouse model of ocular onchocerciasis (river blindness). Infect Immun, 2004. 72(10): p. 5687-92.

64. Pearlman, E., et al., The role of eosinophils and neutrophils in helminthinduced keratitis. Invest Ophthalmol Vis Sci, 1998. 39(7): p. 1176-82.

65. Gallin, M., et al., Cell-mediated immune responses in human infection with Onchocerca volvulus. J Immunol, 1988. 140(6): p. 1999-2007.

66. Song, P.I., et al., The expression of functional LPS receptor proteins CD14 and toll-like receptor 4 in human corneal cells. Invest Ophthalmol Vis Sci, 2001. 42(12): p. 2867-77.

67. Kumar, A., J. Zhang, and F.S. Yu, Innate immune response of corneal epithelial cells to Staphylococcus aureus infection: role of peptidoglycan in stimulating proinflammatory cytokine secretion. Invest Ophthalmol Vis Sci, 2004. 45(10): p. 3513-22. 
68. Ueta, M., et al., Intracellularly expressed TLR2s and TLR4s contribution to an immunosilent environment at the ocular mucosal epithelium. J Immunol, 2004. 173(5): p. 3337-47.

69. Ueta, M., et al., Triggering of TLR3 by polyl:C in human corneal epithelial cells to induce inflammatory cytokines. Biochem Biophys Res Commun, 2005. 331(1): p. 285-94.

70. Ueta, M., et al., Spontaneous ocular surface inflammation and goblet cell disappearance in I kappa B zeta gene-disrupted mice. Invest Ophthalmol Vis Sci, 2005. 46(2): p. 579-88.

71. Zhang, J., et al., Toll-like receptor 5-mediated corneal epithelial inflammatory responses to Pseudomonas aeruginosa flagellin. Invest Ophthalmol Vis Sci, 2003. 44(10): p. 4247-54.

72. $\mathrm{Li}, \mathrm{J}$,, et al., On-line 3-dimensional confocal imaging in vivo. Invest Ophthalmol Vis Sci, 2000. 41(10): p. 2945-53.

73. Takeda, K., T. Kaisho, and S. Akira, Toll-like receptors. Annu Rev Immunol, 2003. 21: p. 335-76.

74. Poltorak, A., et al., Defective LPS signaling in C3H/HeJ and C57BL/10ScCr mice: mutations in TIr4 gene. Science, 1998. 282(5396): p. 2085-8.

75. Taylor, M.J., Wolbachia endosymbiotic bacteria of filarial nematodes. A new insight into disease pathogenesis and control. Arch Med Res, 2002. 33(4): p. 422-4.

76. Nutman, T.B., Lymphatic filariasis: new insights and prospects for control. Curr Opin Infect Dis, 2001. 14(5): p. 539-46.

77. Ottesen, E.A., Immune responsiveness and the pathogenesis of human onchocerciasis. J Infect Dis, 1995. 171(3): p. 659-71.

78. Bradford, M.M., A rapid and sensitive method for the quantitation of microgram quantities of protein utilizing the principle of protein-dye binding. Anal Biochem, 1976. 72: p. 248-54.

79. Tang, Q. and R.L. Hendricks, Interferon gamma regulates platelet endothelial cell adhesion molecule 1 expression and neutrophil infiltration into herpes simplex virus-infected mouse corneas. J Exp Med, 1996. 184(4): p. 1435-47. 
80. Wu, M., et al., Phylogenomics of the reproductive parasite Wolbachia pipientis wMel: a streamlined genome overrun by mobile genetic elements. PLoS Biol, 2004. 2(3): p. E69.

81. Brattig, N.W., D.W. Buttner, and A. Hoerauf, Neutrophil accumulation around Onchocerca worms and chemotaxis of neutrophils are dependent on Wolbachia endobacteria. Microbes Infect, 2001. 3(6): p. 439-46.

82. Ackerman, S.J., et al., Eosinophilia and elevated serum levels of eosinophil major basic protein and Charcot-Leyden crystal protein (lysophospholipase) after treatment of patients with Bancroft's filariasis. J Immunol, 1981. 127(3): p. 1093-8.

83. Pearlman, E., Immunopathology of onchocerciasis: a role for eosinophils in onchocercal dermatitis and keratitis. Chem Immunol, 1997. 66: p. 26-40.

84. Hoerauf, A., et al., Onchocerciasis. Bmj, 2003. 326(7382): p. 207-10.

85. Brattig, N.W., et al., Onchocerca volvulus-exposed persons fail to produce interferon-gamma in response to $O$. volvulus antigen but mount proliferative responses with interleukin-5 and $I L-13$ production that decrease with increasing microfilarial density. J Infect Dis, 2002. 185(8): p. 1148-54.

86. Takeuchi, O. and S. Akira, Toll-like receptors; their physiological role and signal transduction system. Int Immunopharmacol, 2001. 1(4): p. 625-35.

87. Drouet, C., A.N. Shakhov, and C.V. Jongeneel, Enhancers and transcription factors controlling the inducibility of the tumor necrosis factoralpha promoter in primary macrophages. J Immunol, 1991. 147(5): p. 1694700.

88. Gillette-Ferguson, I., et al., Wolbachia- and Onchocerca volvulus-induced keratitis (river blindness) is dependent on myeloid differentiation factor 88. Infect Immun, 2006. 74(4): p. 2442-5.

89. Muller, W.A., et al., PECAM-1 is required for transendothelial migration of leukocytes. J Exp Med, 1993. 178(2): p. 449-60.

90. Wakelin, M.W., et al., An anti-platelet-endothelial cell adhesion molecule-1 antibody inhibits leukocyte extravasation from mesenteric microvessels in vivo by blocking the passage through the basement membrane. J Exp Med, 1996. 184(1): p. 229-39. 
91. Christofidou-Solomidou, M., et al., Neutrophil platelet endothelial cell adhesion molecule-1 participates in neutrophil recruitment at inflammatory sites and is down-regulated after leukocyte extravasation. J Immunol, 1997. 158(10): p. 4872-8.

92. Liao, F., et al., Soluble domain 1 of platelet-endothelial cell adhesion molecule (PECAM) is sufficient to block transendothelial migration in vitro and in vivo. J Exp Med, 1997. 185(7): p. 1349-57.

93. Nakada, M.T., et al., Antibodies against the first Ig-like domain of human platelet endothelial cell adhesion molecule-1 (PECAM-1) that inhibit PECAM-1-dependent homophilic adhesion block in vivo neutrophil recruitment. J Immunol, 2000. 164(1): p. 452-62.

94. Saint Andre, A., et al., The role of endosymbiotic Wolbachia bacteria in the pathogenesis of river blindness. Science, 2002. 295(5561): p. 1892-5.

95. Bazzocchi, C., et al., Immunological role of the endosymbionts of Dirofilaria immitis: the Wolbachia surface protein activates canine neutrophils with production of IL-8. Vet Parasitol, 2003. 117(1-2): p. 73-83.

96. Keiser, P.B., et al., Bacterial endosymbionts of Onchocerca volvulus in the pathogenesis of posttreatment reactions. J Infect Dis, 2002. 185(6): p. 80511.

97. Haarbrink, M., et al., Strong association of interleukin-6 and lipopolysaccharide-binding protein with severity of adverse reactions after diethylcarbamazine treatment of microfilaremic patients. J Infect Dis, 2000. 182(2): p. 564-9.

98. Gutierrez-Pena, E.J., J. Knab, and D.W. Buttner, Neutrophil granule proteins: evidence for the participation in the host reaction to skin microfilariae of Onchocerca volvulus after diethylcarbamazine administration. Parasitology, 1996. 113 (Pt 4): p. 403-14.

99. Volkmann, L., et al., Antibiotic therapy in murine filariasis (Litomosoides sigmodontis): comparative effects of doxycycline and rifampicin on Wolbachia and filarial viability. Trop Med Int Health, 2003. 8(5): p. 392-401.

100. Gallin, M.Y., et al., Human autoantibody to defensin: disease association with hyperreactive onchocerciasis (sowda). J Exp Med, 1995. 182(1): p. 41-7. 
101. Rubio de Kromer, M.T., et al., Detection of a chemotactic factor for neutrophils in extracts of female Onchocerca volvulus. Acta Trop, 1998. 71(1): p. 45-56.

102. Brattig, N.W., Pathogenesis and host responses in human onchocerciasis: impact of Onchocerca filariae and Wolbachia endobacteria. Microbes Infect, 2004. 6(1): p. 113-28.

103. Higazi, T.B., et al., Wolbachia endosymbiont levels in severe and mild strains of Onchocerca volvulus. Mol Biochem Parasitol, 2005. 141(1): p. 109-12.

104. Taylor, M.J., C. Bandi, and A. Hoerauf, Wolbachia bacterial endosymbionts of filarial nematodes. Adv Parasitol, 2005. 60: p. 245-84.

105. Turner, J.D., et al., A randomized, double-blind clinical trial of a 3-week course of doxycycline plus albendazole and ivermectin for the treatment of Wuchereria bancrofti infection. Clin Infect Dis, 2006. 42(8): p. 1081-9.

106. Pfarr, K.M., K. Fischer, and A. Hoerauf, Involvement of Toll-like receptor 4 in the embryogenesis of the rodent filaria Litomosoides sigmodontis. Med Microbiol Immunol (Berl), 2003. 192(1): p. 53-6.

107. The Wold Bank: global partnership to eliminate river blindness.

108. WHO: onchocerciasis www.who.int/tdr/about/products/oncho.htm

109. Little, M.P., et al., Association between microfilarial load and excess mortality in onchocerciasis: an epidemiological study. Lancet, 2004. 363(9420): p. 1514-21.

110. Campbell, W.C., Ivermectin as an antiparasitic agent for use in humans. Annu Rev Microbiol, 1991. 45: p. 445-74.

111. Awadzi, K., et al., The chemotherapy of onchocerciasis XX: ivermectin in combination with albendazole. Trop Med Parasitol, 1995. 46(4): p. 213-20.

112. Alley, W.S., et al., Macrofilaricides and onchocerciasis control, mathematical modelling of the prospects for elimination. BMC Public Health, 2001. 1(1): p. 12.

113. Plaisier, A.P., et al., Irreversible effects of ivermectin on adult parasites in onchocerciasis patients in the Onchocerciasis Control Programme in West Africa. J Infect Dis, 1995. 172(1): p. 204-10. 
114. Ali, M.M., et al., Immunocompetence may be important in the effectiveness of Mectizan (ivermectin) in the treatment of human onchocerciasis. Acta Trop, 2002. 84(1): p. 49-53.

115. Ardelli, B.F. and R.K. Prichard, Identification of variant ABC-transporter genes among Onchocerca volvulus collected from ivermectin-treated and untreated patients in Ghana, West Africa. Ann Trop Med Parasitol, 2004. 98(4): p. 371-84.

116. Ardelli, B.F., S.B. Guerriero, and R.K. Prichard, Genomic organization and effects of ivermectin selection on Onchocerca volvulus P-glycoprotein. Mol Biochem Parasitol, 2005. 143(1): p. 58-66.

117. Ardelli, B.F., S.B. Guerriero, and R.K. Prichard, Characterization of a halfsize ATP-binding cassette transporter gene which may be a useful marker for ivermectin selection in Onchocerca volvulus. Mol Biochem Parasitol, 2006. 145(1): p. 94-100.

118. Ardelli, B.F., S.B. Guerriero, and R.K. Prichard, Ivermectin imposes selection pressure on P-glycoprotein from Onchocerca volvulus: linkage disequilibrium and genotype diversity. Parasitology, 2006. 132(Pt 3): p. 375-86.

119. Eng, J.K. and R.K. Prichard, A comparison of genetic polymorphism in populations of Onchocerca volvulus from untreated-and ivermectin-treated patients. Mol Biochem Parasitol, 2005. 142(2): p. 193-202.

120. Awadzi, K., et al., Thirty-month follow-up of sub-optimal responders to multiple treatments with ivermectin, in two onchocerciasis-endemic foci in Ghana. Ann Trop Med Parasitol, 2004. 98(4): p. 359-70.

121. Wolstenholme, A.J., et al., Drug resistance in veterinary helminths. Trends Parasitol, 2004. 20(10): p. 469-76.

122. Awadzi, K., et al., The safety, tolerability and pharmacokinetics of levamisole alone, levamisole plus ivermectin, and levamisole plus albendazole, and their efficacy against Onchocerca volvulus. Ann Trop Med Parasitol, 2004. 98(6): p. 595-614.

123. Awadzi, K., et al., The safety and efficacy of amocarzine in African onchocerciasis and the influence of ivermectin on the clinical and parasitological response to treatment. Ann Trop Med Parasitol, 1997. 91(3): p. 281-96. 
124. Awadzi, K. and H.M. Gilles, The chemotherapy of onchocerciasis iii. A comparative study of diethylcarbamazide (DEC) and metrifonate. Ann Trop Med Parasitol, 1980. 74(2): p. 199-210.

125. Awadzi, K., Clinical picture and outcome of Serious Adverse Events in the treatment of Onchocerciasis. Filaria J, 2003. 2 Suppl 1: p. S6.

126. Schwab, A.E., et al., Detection of benzimidazole resistance-associated mutations in the filarial nematode Wuchereria bancrofti and evidence for selection by albendazole and ivermectin combination treatment. Am J Trop Med Hyg, 2005. 73(2): p. 234-8.

127. Hoerauf, A., et al., Depletion of wolbachia endobacteria in Onchocerca volvulus by doxycycline and microfilaridermia after ivermectin treatment. Lancet, 2001. 357(9266): p. 1415-6.

128. Taylor, M.J. and A. Hoerauf, $A$ new approach to the treatment of filariasis. Curr Opin Infect Dis, 2001. 14(6): p. 727-31.

129. Hoerauf, A., et al., Doxycycline in the treatment of human onchocerciasis: Kinetics of Wolbachia endobacteria reduction and of inhibition of embryogenesis in female Onchocerca worms. Microbes Infect, 2003. 5(4): p. 261-73.

130. Hoerauf, A., et al., Doxycycline as a novel strategy against bancroftian filariasis-depletion of Wolbachia endosymbionts from Wuchereria bancrofti and stop of microfilaria production. Med Microbiol Immunol, 2003. 192(4): p. 211-6.

131. Taylor, M.J., et al., Macrofilaricidal activity after doxycycline treatment of Wuchereria bancrofti: a double-blind, randomised placebo-controlled trial. Lancet, 2005. 365(9477): p. 2116-21.

132. Hoerauf, A., et al., Call to consolidate achievements for onchocerciasis and lymphatic filariasis control. Trends Parasitol, 2001. 17(12): p. 566-7. 



\section{ACKNOLEDGEMENTS}

I thank Professor Eric Pearlman for his help and advice in the research and publication of this study.

I thank Professor Dr. med. Matthias Frosch and the Studienstiftung des Deutschen Volkes for their great support of this thesis.

Thanks to Helen Running for proof reading this work.

My thanks to: my parents Achim und Bettina von Saint Andre - von Arnim; my aunt Almut von Welck; my husband and son Seth and Achim Bagan. 



\section{CURRICULUM VITAE}

Amélie von Saint André - von Arnim

\section{EDUCATION}

June 2005 - present

Boston Combined Residency Program in Pediatrics, Boston, MA, USA

Currently third year resident

Nov 1998 - May $2004 \quad$ Julius - Maximilians - University Würzburg School of Medicine, Würzburg, Germany

Graduation in May 2004

Aug 1996 - Aug 1998 Semmelweis University, Budapest, Hungary

Preclinical years

Preliminary Medical Examination, August 1998

Aug 1993 - May $1995 \quad$ United World College of the Atlantic, Wales,

Great Britain

International Baccalaureate, May 1995

Aug 1992 - July 1993 Liceo scientifico Golgi, Breno, Italy

One year Rotary exchange program.

Aug 1988 - July 1992 Altes Gymnasium Oldenburg, Oldenburg, Niedersachsen

RESEARCH

Sept 2000 - May $2001 \quad$ Research Assistant

University Hospitals of Cleveland and Case Western

Reserve University, Cleveland, $\mathrm{OH}$

Research on the pathogenesis of River Blindness

PUBLICATIONS

Science, March 2002, Amélie v. Saint André et al

Vol. 295, pp. 1892-1895 "The Role of Endosymbiotic Wolbachia Bacteria in the Pathogenesis of River Blindness"

HONORS \& AWARDS
$2000-2004$
German National Scholarship. Merit based award by the Studienstiftung des deutschen Volkes
$1993-1995$
Full Tuition Merit Scholarship. United World College of the Atlantic, Wales, Great Britain
$1992-1993$
Exchange Scholarship. Rotary International Award for a one year exchange program at Liceo Scientifico Golgi, Breno, Italy 